OPEN ACCESS

Edited by:

Bimal Malhotra,

Pfizer, United States

Reviewed by:

Nazareno Paolocci, Johns Hopkins University,

United States

Joachim Neumann Institut für Pharmakologie und

Toxikologie, Germany

*Correspondence:

Hongxin Wang

hongxinwang@jzmu.edu.cn

Futian Tang

tangft@163.com

Specialty section:

This article was submitted to Cardiovascular and Smooth Muscle

Pharmacology,

a section of the journal

Frontiers in Pharmacology

Received: 28 April 2018 Accepted: 24 September 2018

Published: 11 October 2018

Citation:

Lu M, Leng B, He X, Zhang Z, Wang $H$ and Tang $F$ (2018) Calcium Sensing Receptor-Related Pathway

Contributes to Cardiac Injury and the Mechanism of Astragaloside IV on Cardioprotection.

Front. Pharmacol. 9:1163.

doi: 10.3389/fphar.2018.01163

\section{Calcium Sensing Receptor-Related Pathway Contributes to Cardiac Injury and the Mechanism of Astragaloside IV on Cardioprotection}

\author{
Meili Lu', Bin Leng ${ }^{1}$, Xin $\mathrm{He}^{2}$, Zhen Zhang ${ }^{1}$, Hongxin Wang ${ }^{1 *}$ and Futian Tang ${ }^{1 *}$ \\ ${ }^{1}$ Key Laboratory of Cardiovascular and Cerebrovascular Drug Research of Liaoning Province, Jinzhou Medical University, \\ Jinzhou, China, ${ }^{2}$ Internal Medicine-Cardiovascular Department, The First Affiliated Hospital of Jinzhou Medical University, \\ Jinzhou, China
}

Activation of calcium sensing receptor (CaSR) contributes to cardiac injury, but the underlying mechanism has not yet been examined. Astragaloside IV (AsIV) was previously reported to exhibit protective effects against various myocardial injuries. The aim of the present study was to investigate the underlying mechanism of CaSR in cardiac hypertrophy and apoptosis and to evaluate whether the protective effect of AsIV against myocardial injury is associated with CaSR and its related signaling pathway. In vivo and in vitro myocardial injury was induced by isoproterenol (Iso) or $\mathrm{GdCl}_{3}$ (a CaSR agonist) in rats and heart H9C2 cells. Cardiac cell hypertrophy, apoptosis, function, Mitochondrial Membrane Potential (MMP), mitochondrial ultrastructure, and $\left[\mathrm{Ca}^{2+}\right]_{\mathrm{i}}$, as well as the protein expression of CaSR, calcium/calmodulin-dependent protein kinase II (CaMKII), calcineurin (CaN), sarcoplasmic reticulum $\mathrm{Ca}^{2+}$-ATPase2a (SERCA2a), and the inositol 1,4,5-trisphosphate receptor (IP3R), were measured in vivo and/or in vitro. The results showed that AsIV attenuated cardiac hypertrophy and apoptosis and attenuated impairments in cardiac function, mitochondrial structure, and MMP induced by Iso or $\mathrm{GdCl}_{3}$ in rat myocardial tissue and H9C2 cells. Importantly, AsIV treatment inhibited the enhancement of $\left[\mathrm{Ca}^{2+}\right]_{i}$ and CaSR expression induced by Iso or $\mathrm{GdCl}_{3}$, an effect similar to that of the CaSR antagonist NPS2143. In addition, AsIV treatment repressed CaSR, CaMKII, and CaN activation and inhibited NFAT3 nuclear translocation. Mechanistic analysis using lentivirus infection showed that CaSR overexpression activated the CaMKII and CaN signaling pathways and that this response was enhanced by Iso. The results suggested that CaSR-mediated changes in $\left[\mathrm{Ca}^{2+}\right]_{i}$ and CaMKII and CaN signaling pathways contribute to cardiac hypertrophy and apoptosis and are involved in the protective effect of astragaloside IV against cardiac injury.

Keywords: astragaloside IV, calcium sensing receptor, cardiac hypertrophy, apoptosis, calcineurin 


\section{INTRODUCTION}

Heart failure is the end stage of many cardiovascular diseases and is a major public health problem. Cardiomyocyte apoptosis and hypertrophy contribute to the progression of heart failure. During apoptosis, the loss of hypertrophic cardiomyocytes exacerbates the myocardial contractility, aggravates myocardial fibrosis, reduces myocardial contractility, and ultimately contributes to heart failure ( $\mathrm{Li}$ et al., 2009; Xu et al., 2011). Hypertrophic cardiomyocyte impairs the coordination of myocardial contraction and predisposes individuals to heart failure and sudden death. Hypertrophy of cardiomyocytes induced by $\beta$-adrenergic receptor stimulation accelerates the progress from compensated hypertrophy to heart failure, and the mechanisms are related to calcium homeostasis and to complex interactions in intracellular signaling pathways (Dewenter et al., 2017; Khalilimeybodi et al., 2017; Kim et al., 2017).

Calcium sensing receptor (CaSR) is a member of the $G$ Protein-Coupled Receptors (GPCRs) superfamily, that was found to be expressed in the hearts and neonatal rat cardiomyocyte (Sun et al., 2006; Tfelt-Hansen et al., 2006) and is involved in systemic homeostasis of calcium and metal ions. Moreover, the CaSR in the cardiovascular system plays an important role in pulmonary hypertension, atherosclerosis, and ischemia/reperfusion injury (Li et al., 2012; Smith et al., 2016). Previous studies have shown that CaSR is involved in myocardial ischemia reperfusion injury through MAPK activation, phospho-PKC $\delta$ translocation to the mitochondria, calcium overload, caspase- 3 activity, and Fas death receptor pathways (Jiang et al., 2008; Lu et al., 2010; Zheng et al., 2011). Subsequent studies have revealed the expression patterns and the critical role of CaSR in cardiac hypertrophy and apoptosis and have demonstrated that CaSR induces myocardial apoptosis through a mitochondrial dynamicsmediated apoptotic pathway in hypertensive hearts, aggravates myocardial hypertrophy through activation of autophagy, and induces $\mathrm{Ca}^{2+}$ release from the sarcoplasmic reticulum into the mitochondria in a rat model of heart failure ( $\mathrm{Lu}$ et al., 2013; Hong et al., 2017). Although some progress has been made in understanding the role of CaSR in the cardiovascular system, the exact mechanism by which CaSR participates in cardiac hypertrophy and the downstream signaling pathway is not fully understood.

Astragaloside IV (AsIV), a purified small molecular saponin, is one of the major and active components in Astragalus membranaceus. Several studies have indicated that it has a wide spectrum of pharmacological effects including anti-inflammatory effects, antioxidative effects, regulatory effects on energy metabolism, and anti-apoptotic effects (Guo et al., 2016; Jiang et al., 2017; Li et al., 2017, 2018). These beneficial pharmacological and biochemical activities confer AsIV a potential therapeutic role in cardiovascular diseases. For instance, AsIV could improve cardiac function, alleviate ventricular remodeling via stimulating fatty acid $\beta$-oxidation, improve mitochondrial function and regulate $\mathrm{Ca}^{2+}$ homeostasis in rat model of heart failure (Tang et al., 2018). In addition, AsIV has been report to reduce the severity of myocarditis, attenuate cardiac inflammation, attenuate cardiac fibrosis induced by coxsackievirus B3 (CVB3), an effect that was mediated by inhibition of nuclear factorkappaB (NF-kB) and transforming growth factor- $\beta 1$ (TGF- $\beta 1$ ) signaling (Gui et al., 2015). Consistent with the results of these studies, we previously demonstrated a cardioprotective role of AsIV in Iso-induced cardiac hypertrophy that was at least partly attributed to the inhibition of calpain-1 activation and the TLR4/NF-אB signaling pathway (Yang et al., 2013; Mei et al., 2015). However, the effect of AsIV on $\mathrm{Ca}^{2+}$ homeostasis and CaSR in Iso-induced apoptosis and hypertrophy remains poorly understood. Therefore, we conducted the present study to test whether CaSR contributes to $\mathrm{Ca}^{2+}$ homeostasis and the $\mathrm{Ca}^{2+}$ mediated hypertrophic and apoptotic signaling pathway and whether this molecular mechanism is involved in the protective effect of AsIV against Iso induced myocardial injury.

\section{MATERIALS AND METHODS}

\section{Materials}

AsIV was obtained from Nanjin Jingzhu Biotechnology Company (purity >98\% measured by HPLC; Nanjing, China). Iso(I5627), $\mathrm{GdCl}_{3}$ (G7532), and 2-APB(D9754), Dimethyl Sulfoxide (DMSO) and rhodamine-labeled phalloidin were purchased from SigmaAldrich (St. Louis, MO, United States). NPS2143 (S2633) was purchased from Selleck Chemicals (Houston, TX, United States). Antibodies against CaMKII(AB11287), SERCA2a (AB41825), and IP3R(AB12382) were purchased from Absci (Baltimore, MD, United States). Antibodies against NFAT-3 (ab99431), GATA-4 (ab84593), Bcl-2 (ab196495), and Bax(ab5313) were purchased from Abcam (Cambridge, MA, United States). Antibodies against CaN (13198-2-AP), CaSR (19125-1-AP), and $\beta$-actin (60008-1-Ig) were purchased from Proteintech Biotechnology (Wuhan, China). Nuclear and mitochondrial extraction kits were purchased from Vazyme Biotech Co., Ltd. (Nanjing, China). A 5,5',6,6'-tetrachloro-1,1',3,3'-tetraethylbenzimidazol-carbocyanine iodide (JC-1) kit and Fluo-4 were obtained from the Beyotime Institute of Biotechnology (Nanjing, China). A terminal deoxynu-cleotidyl Transferase-Mediated dUTP Nick-End Labeling (TUNEL) kit was purchased from Roche (Darmstadt, Germany). TRIzol reagent, ANP and BNP primers were obtained from TaKaRa Biotechnology Co. (Dalian, China).

\section{Animal Experiments}

The experimental protocols were approved by the Committee of Jinzhou Medical University for the Use of Experimental Animals for Research and Teaching. 60 Male Sprague-Dawley rats, weighting 220 to $250 \mathrm{~g}$, were purchased from the Animal Center of Jinzhou Medical University (Certificate No. SCXK 2017-0003). The rats were housed under a $12 \mathrm{~h}: 12 \mathrm{~h}$ light:dark cycle at $22 \pm 2^{\circ} \mathrm{C}$ with $65-69 \%$ humidity and received a standard diet and water ad libitum. After 3 days preadaptation the rats were randomly divided into 6 groups $(n=10)$ : (1) the Con group; (2) the Iso (10 mg/kg/d, i.p.) group; (3) the Iso + AsIV group; (4) the NPS2143 (1 mg/kg/d, i.p.) + Iso group; (5)the $\mathrm{GdCl}_{3}$ $\left(10 \mathrm{mg} / \mathrm{kg} / \mathrm{d}\right.$, i.p.) group; and (6) the $\mathrm{GdCl}_{3}+\mathrm{AsIV}$ group. Rats in the AsIV group were gavaged with $80 \mathrm{mg} / \mathrm{kg}$ of AsIV 
suspended in $0.5 \%$ sodium carboxymethylcellulose (CMC) 1 day before Iso and $\mathrm{GdCl}_{3}$ administration, and all the drugs were given for 14 days. The dosages of $\mathrm{GdCl}_{3}$ and NPS2143 were selected according to previous studies (Zhao et al., 2011; Yamamura et al., 2012).

\section{Heart Weight Index Measurement}

All animals were weighted and anesthetized with $20 \%$ urethane $(0.5 \mathrm{ml} / 100 \mathrm{~g}$, i.p.) at the end of the experiment. Then, the hearts of the rats were immediately harvested, rinsed in icecold $0.9 \% \mathrm{NaCl}$ solution, dissected and weighed. The heartweight index (the ratio of the heart weight to the body weight, $\mathrm{HW} / \mathrm{BW}$ ) and the left ventricle-weight index (the ratio of the left ventricular weight to the body weight, $\mathrm{LVW} / \mathrm{BW}$ ) were calculated separately. The heart tissues were weighed on a balance and then immediately put into liquid nitrogen or $4 \%$ formaldehyde for the next experiments.

\section{Electron Microscopy Analysis}

Rats were perfused with $2.5 \%$ glutaraldehyde, and the heart tissues were taken, cut into $<1 \mathrm{~mm}^{3}$ pieces, and fixed in $3 \%$ glutaraldehyde for $4 \mathrm{~h}$ and $1 \%$ osmic acid for $2 \mathrm{~h}$. Then, the heart tissues were dehydrated with ethanol and propylene oxide, embedded in Epon-812, cut into ultrathin sections, stained with uranyl acetate and lead citrate and observed with a Philips CM 120 electron microscope (Amsterdam, Holland).

\section{HE and TUNEL Staining}

Hearts tissues were fixed in $4 \%$ formaldehyde for $24 \mathrm{~h}$ and were then embedded in paraffin, cut into $5 \mu \mathrm{m}$ sections, and stained with Hematoxylin-Eosin (HE). The TUNEL assay was performed according to the manufacturer's protocol. The index of apoptosis was expressed as the number of positively stained apoptotic cardiomyocytes / the total number of cardiomyocytes counted $\times 100 \%$.

\section{Echocardiography}

Heart function was evaluated at the end of the experiment using a Siemens Acuson SC2000 high-frequency ultrasound system (Siemens, Inc., Berlin, Germany). The rats were anesthetized with inhaled isoflurane, and the LV Ejection Fraction (LVEF), LV fractional shortening (LVFS), and LV Internal Diastolic Diameter (LVIDd) were measured and analyzed using the M-mode.

\section{Cell Culture and Estimation of Cell Volume}

H9C2 cells were obtained from Wuhan Boster Biotech Company (Wuhan, China) and propagated in Dulbecco's Modified Eagle's Medium (DMEM, Gibco) supplemented with 10\% fetal bovine serum (FBS, HyClone) and 1\% penicillin-streptomycin (Invitrogen). The cells were fixed with $4 \%$ paraformaldehyde at room temperature for $30 \mathrm{~min}$, washed with PBS, and then treated with $0.5 \mu \mathrm{M}$ rhodamine-labeled phalloidin for $30 \mathrm{~min}$ and $0.5 \mu \mathrm{M}$ DAPI for $10 \mathrm{~min}$. Then, the cells were examined and photographed using a fluorescence microscope.

\section{Lentiviral Overexpression of CaSR in H9C2 Cells}

Rat H9C2 cells $\left(5 \times 10^{4} / \mathrm{ml}\right)$ were prepared and infected at a Multiplicity of Infection (MOI) of 50 with control or CaSRoverexpressing lentiviruses (Shanghai GeneChem) for $24 \mathrm{~h}$ at $37^{\circ} \mathrm{C}$ in the presence of $10 \mathrm{mg} / \mathrm{ml}$ polybrene. The $\mathrm{H} 9 \mathrm{C} 2$ cells were then washed and cultured in fresh medium for $24 \mathrm{~h}$ for further analysis.

\section{ANP and BNP Measurement}

Total RNA was extracted from H9C2 cells using TRIzol reagent according to the manufacturer's protocol. The total RNA concentration was determined based on the absorption at $260 \mathrm{~nm}$, and the purity was determined according to the A260/A280 ratios. The same amount of total RNA $(2 \mu \mathrm{g})$ was used from each sample. RNA was reverse transcribed using AMV reverse transcriptase with random hexamers for $50 \mathrm{~min}$ at $42^{\circ} \mathrm{C}$. The cDNA was denatured at $95^{\circ} \mathrm{C}$ for $5 \mathrm{~s}$ followed by $40 \mathrm{PCR}$ cycles using ABI 7500 fast real time PCR system (Foster, CA, United States). The relative level of mRNA was calculated by the comparative $\mathrm{C}_{\mathrm{T}}$ method with GAPDH mRNA as the invariant control.

\section{Apoptosis Assays}

Apoptosis was detected using an annexin V-FITC apoptosis detection kit according to the manufacturer's instructions. Briefly, H9C2 cells were seeded in a $25 \mathrm{~cm}^{2}$ flask and incubated with the indicated drugs for $24 \mathrm{~h}$. The cells were harvested, washed twice with prechilled PBS and centrifuged at $2000 \mathrm{rpm}$ for $5 \mathrm{~min}$ followed by staining with annexin V-FITC using an assay kit. The data were analyzed using the Bioconsort software (United States).

\section{Fluo-4/AM Measurements of Intracellular $\left[\mathrm{Ca}^{2+}\right]_{\mathrm{i}}$}

H9C2 cells were cultured in confocal culture dishes and then loaded with $5 \mu \mathrm{M}$ Fluo-4/AM for $30 \mathrm{~min}$ at $37^{\circ} \mathrm{C}$ in the dark. The H9C2 cells were then washed twice with $\mathrm{Ca}^{2+}$-free PBS to remove the extracellular Fluo-4/AM and incubated further in DMEM. Changes in $\left[\mathrm{Ca}^{2+}\right]_{\mathrm{i}}$ were measured by the fluorescence intensity induced by Fluo-4 in H9C2 cells recorded for 5 min using Leica TCS SP5II laser confocal scanning microscopy (Wetzlar, Germany).

\section{Measurement of Mitochondrial Membrane Potential (MMP)}

Mitochondrial Membrane Potential (MMP) was determined by JC-1 staining. After the different treatments, H9C2 cells were incubated for $20 \mathrm{~min}$ in the presence of $2 \mu \mathrm{M} \mathrm{JC}-1$ at $37^{\circ} \mathrm{C}$. Then, the cells were washed twice with JC-1 buffer solution. Thereafter, labeled cells were analyzed and quantified by Leica DMI3000B fluorescence microscope (Wetzlar, Germany).

\section{Immunofluorescence}

H9C2 cells were fixed in 4\% paraformaldehyde, permeabilized with $0.5 \%$ Triton X-100, and blocked with 5\% BSA for $30 \mathrm{~min}$ at room temperature. The cells were then incubated with a CaSR 
A

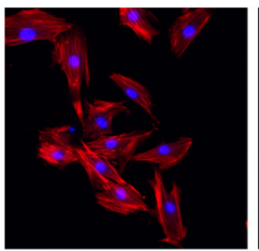

Con

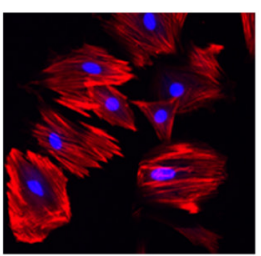

Iso

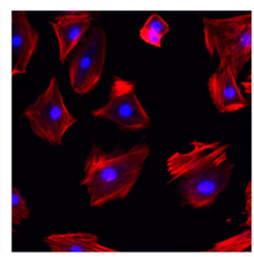

Iso+AsIV

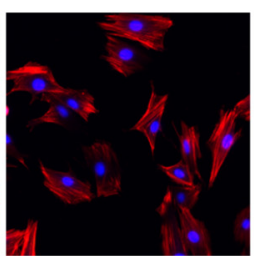

Iso+NPS2143

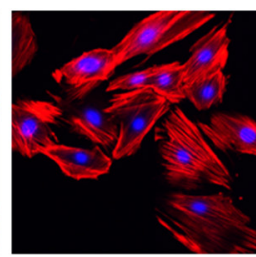

$\mathrm{GdCl}_{3}$

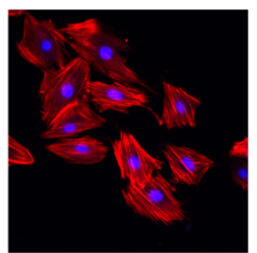

$\mathrm{GdCl}_{3}+\mathrm{AsIV}$
B

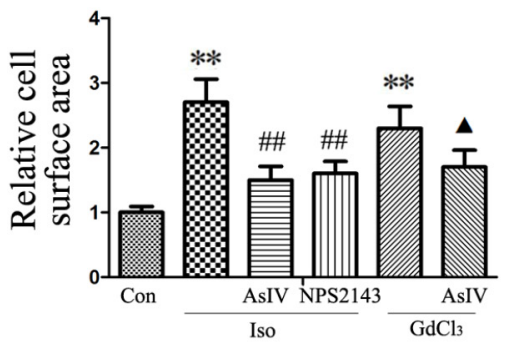

C

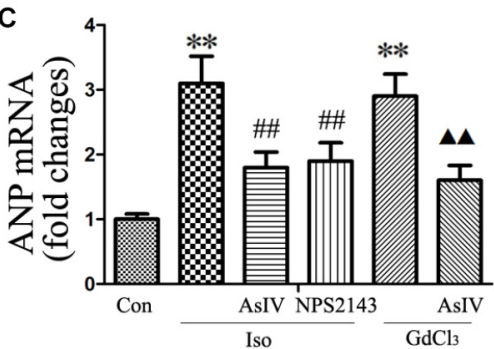

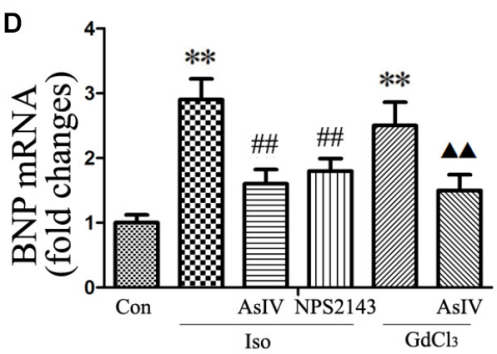

FIGURE 1 | AsIV inhibited H9C2 cell hypertrophy induced by Iso or $\mathrm{GdCl}_{3}$. (A) H9C2 cells were stained with rhodamine-labeled phalloidin and DAPI and then examined and photographed using a fluorescence microscope (magnification $\times 200$ ). (B) Cell surface area was measured and analyzed with LAS Software (V4.3) (Leica, Germany). The bars represent the cellular size relative to that of the controls. (C,D) mRNA expression of ANP and BNP. The data are expressed as the means \pm SEM, $n=4$. ${ }^{* *} P<0.01$ vs. the Con group; ${ }^{\#} P<0.01$ vs. the Iso group; and $\boldsymbol{\Delta} P<0.05, \boldsymbol{\Delta} \boldsymbol{\Delta} P<0.01$ vs. the GdCl 3 group.

A

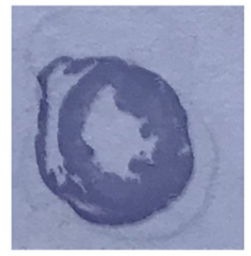

B

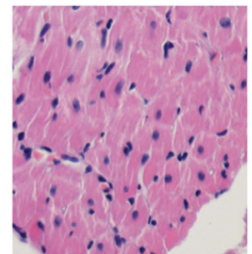

Con
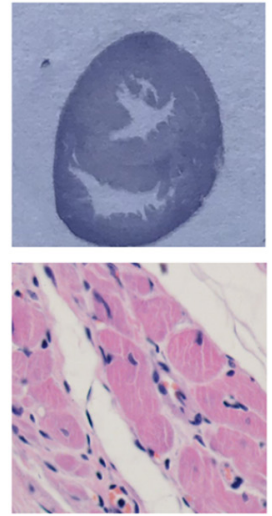

Iso
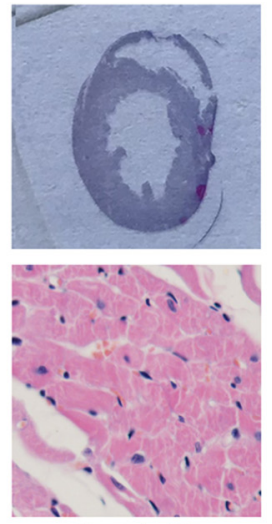

Iso+AsIV
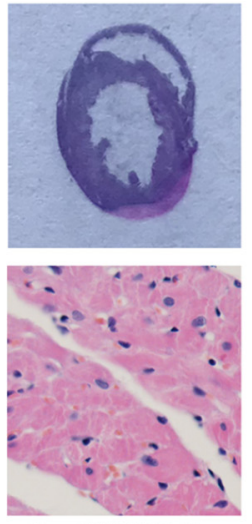

Iso+NPS2143
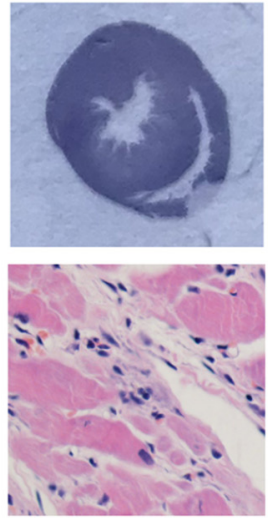

$\mathrm{GdCl}_{3}$
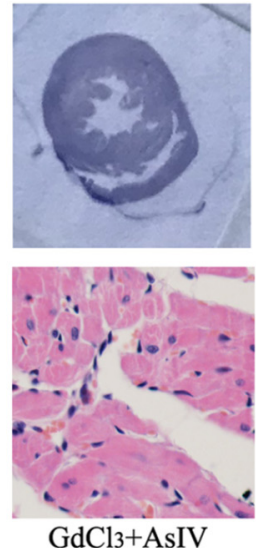

$\mathrm{GdCl}_{3}+\mathrm{AsIV}$
C

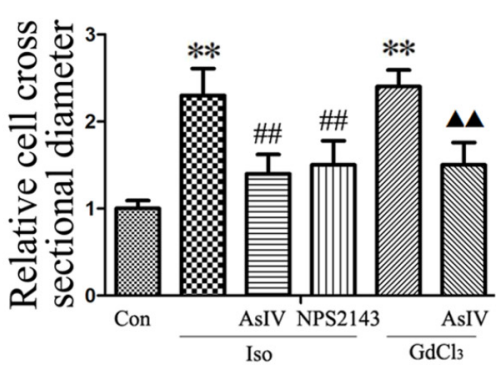

D

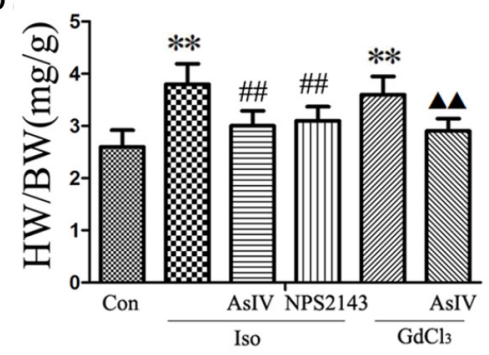

E

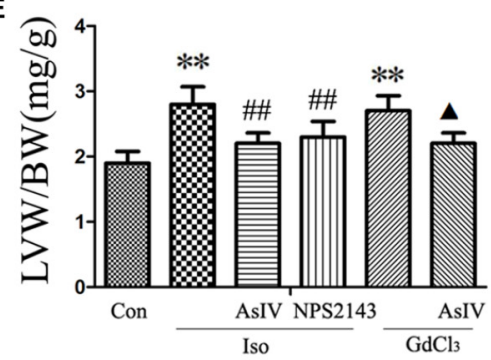

FIGURE 2 | AsIV inhibited cardiac hypertrophy induced by Iso or $\mathrm{GdCl}_{3}$ in rats. (A) Left ventricular tissue section stained with H\&E. (B) Representative example of H\&E staining of heart tissue. (C) Statistical data on cell surface area shown by H\&E staining and measured and analyzed with LAS Software. (D,E) Ratios of heart weight/body weight (HW/BW) and left ventricular weight/body weight (LWW/BW). Data are expressed as the means \pm SEM, $n=4$ for A-C; $n=8$ for D and E. ${ }^{* *} P<0.01$ vs. the Con group; $\# \#<0.01$ vs. the Iso group; and $\mathbf{\Delta} P<0.05, \boldsymbol{\Delta} \mathbf{\Delta} P<0.01$ vs. the $\mathrm{GdCl}_{3}$ group. 
antibody $(1: 100)$ overnight at $4^{\circ} \mathrm{C}$. The next day, the cells were washed three times and incubated with fluorescein isothiocyanate (FITC)-conjugated goat anti-rabbit secondary antibody for $1 \mathrm{~h}$ at $37^{\circ} \mathrm{C}$ in the dark. After being washed with PBS, the cells were treated with $0.5 \mu \mathrm{M}$ rhodamine-labeled phalloidin for $30 \mathrm{~min}$ and $0.5 \mu \mathrm{M}$ DAPI for $10 \mathrm{~min}$. Then, the cells were examined and photographed using Leica DMI3000B fluorescence microscope (Wetzlar, Germany).

\section{Preparation of Protein Extracts and Western Blot Analysis}

Nuclear and mitochondrial proteins were extracted from heart tissues and H9C2 cells using a nuclear and mitochondrial extraction kit according to the manufacturer's instructions. The protein concentration was determined by the BCA method. After boiling the samples for $5 \mathrm{~min}$, the protein samples were fractionated by SDS-PAGE (10-12\% polyacrylamide gels), transferred to Polyvinylidene Fluoride (PVDF) membranes (Millipore, Bedford, MA) and blocked with 1\% BSA for $2 \mathrm{~h}$. The membranes were incubated with primary antibodies for CaSR, CaMKII, CaN, NFAT-3, GATA4, Bcl-2, Bax, and $\beta$-actin at room temperature for $1.5 \mathrm{~h}$. Detection was performed with enhanced chemiluminescence reagents. The results were analyzed with Quantity One software (Bio-Rad Laboratories, Hercules).

\section{Statistics}

All data are expressed as the means \pm SEM. SPSS 17.0 software (Chicago, IL, United States) was used to analyze all the data. Statistical analysis was performed using one-way ANOVA followed by Bonferroni's test. $P<0.05$ was considered statistically significant.

\section{RESULTS}

\section{AsIV Inhibited Cardiac Hypertrophy and Corrected Cardiac Dysfunction Induced by Iso or $\mathrm{GdCl}_{3}$}

To investigate the inhibitory effect of AsIV on cardiac hypertrophy, we created a hypertrophy model using Iso or $\mathrm{GdCl}_{3}$. The results showed that activation of CaSR with $\mathrm{GdCl}_{3}(30 \mu \mathrm{M})$ induced cardiac hypertrophy, as indicated by the increased cell surface area (Figures 1A,B) and increased mRNA expression of
ANP and BNP in H9C2 cells (Figures 1C,D), effects that were similar to the effects of Iso $(10 \mu \mathrm{M})$. Injecting rats with Iso or $\mathrm{GdCl}_{3}$ for 14 days induced cardiac hypertrophy, as indicated by the increased HW/BW and LVW/BW ratios (Figures 2D,E), the cardiomyocyte cross-sectional diameter and ventricular wall thickness (Figures 2A-C). The present study showed that $100 \mu \mathrm{M}$ AsIV inhibited Iso-induced cardiac hypertrophy similarly to the CaSR antagonist NPS2143 (1 $\mu \mathrm{M})$. In addition, the combination of AsIV with $\mathrm{GdCl}_{3}$ also ameliorated cardiac hypertrophy induced by $\mathrm{GdCl}_{3}$. Furthermore, cardiac function was evaluated individually by estimation of the LVEF, LVFS, and LVIDd through echocardiography. Compared with the parameters in the control group, rats injected with Iso or $\mathrm{GdCl}_{3}$ for 14 days resulted in decreased LVEF and LVFS and increased LVIDd, indicating hypertrophy in the decompensation phase, and cardiac function was impaired. However, the combination of AsIV with Iso or $\mathrm{GdCl}_{3}$ improved cardiac function, as evidenced by increased LVEF and LVFS and decreased LVIDd (Table 1).

\section{AsIV Inhibited Apoptosis in Hypertrophied Heart Tissue and Cell}

Hypertrophic cardiomyocytes are more susceptible to apoptosis, promoting the transformation from hypertrophy to heart failure. To further explore the protective effect of AsIV against cardiac injury, we examined the apoptotic rate and the protein expression of Bax and Bcl-2. The results showed that AsIV decreased the number of Annexin-V/PI positive cells in the H9C2 cells (Figures $3 \mathrm{~A}, \mathbf{B}$ ), reduced the number of TUNELpositive cells in heart tissue (Figures 3E,F), upregulated Bcl2 expression and downregulated Bax expression compared with these parameters in myocardial tissue (Figures 3G,H) and $\mathrm{H} 9 \mathrm{C} 2$ cells (Figures 3C,D) treated with Iso or $\mathrm{GdCl}_{3}$ alone, and NPS2143 had anti-apoptotic effects similar to those of AsIV.

\section{AsIV Improved Mitochondrial Ultrastructure and MMP Dissipation}

Mitochondrial Membrane Potential (MMP) dissipation and cytochrome $\mathrm{c}$ release from the mitochondria to the cytoplasm prove the involvement of the mitochondrial apoptotic pathway in heart failure. The present study observed the effect of AsIV on Iso or $\mathrm{GdCl}_{3}$-induced MMP dissipation and cytochrome c release. In live cells, the mitochondria appear red due

TABLE 1 | Parameters of cardiac function in rats.

\begin{tabular}{lccc}
\hline Group & LVEF (\%) & LVFS (\%) & LVIDd (mm) \\
\hline Con & $77.45 \pm 4.45$ & $33.38 \pm 3.84$ & $6.18 \pm 0.43$ \\
Iso & $57.73 \pm 5.44^{* *}$ & $20.10 \pm 3.76^{* *}$ & $8.10 \pm 0.36^{* *}$ \\
Iso + AsIV & $69.50 \pm 5.63^{\# \#}$ & $30.68 \pm 4.37^{\# \#}$ & $6.80 \pm 0.34^{\# \#}$ \\
Iso + NPS2143 & $66.35 \pm 5.65^{\#}$ & $28.95 \pm 2.38^{\# \#}$ & $6.95 \pm 0.39^{\# \#}$ \\
$\mathrm{GdCl}_{3}$ & $59.33 \pm 7.01^{* *}$ & $21.45 \pm 3.14^{* *}$ & $7.63 \pm 0.57^{* *}$ \\
$\mathrm{GdCl}_{3}+$ AsIV & $70.67 \pm 3.93^{\mathbf{\Lambda}}$ & $30.55 \pm 3.70^{\mathbf{\Delta}}$ & $6.92 \pm 0.52^{\mathbf{\Lambda}} \mathbf{4}$ \\
\hline
\end{tabular}

LVEF, left ventricular ejection fraction; LVFS, left ventricular fractional shortening; and LVIDd, left ventricular internal diastolic diameter. $n=4$ rats for each group. **P $<0.01$ vs. the Con group; ${ }^{\#} P<0.01$, ${ }^{\#} P<0.05$ vs. the Iso group; and. $\mathbf{\Delta} P<0.01$ vs. the $\mathrm{GdCl}_{3}$ group. 
A

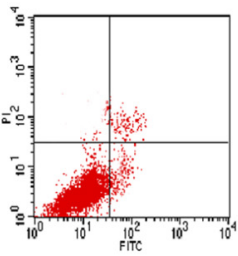

Con

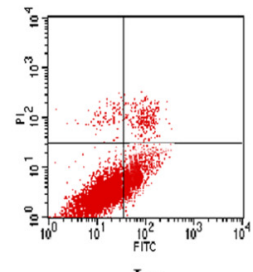

Iso

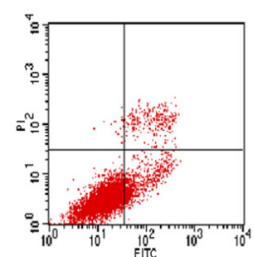

Iso+AsIV

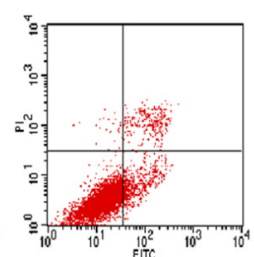

Iso+NPS2143

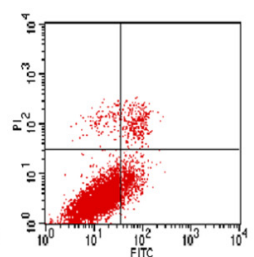

$\mathrm{GdCl}_{3}$

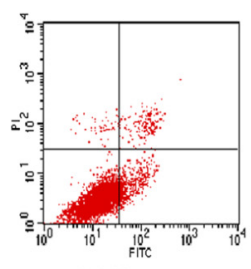

$\mathrm{GdCl}_{3}+\mathrm{AsIV}$
B

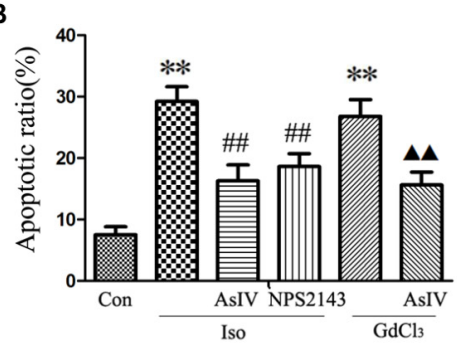

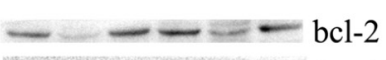

C

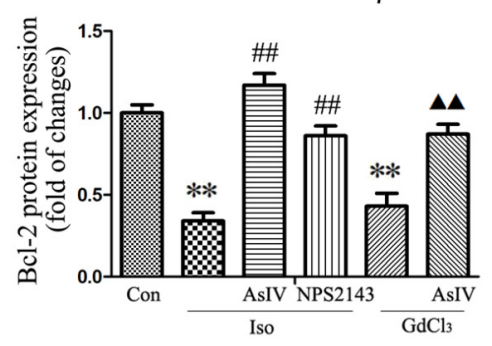

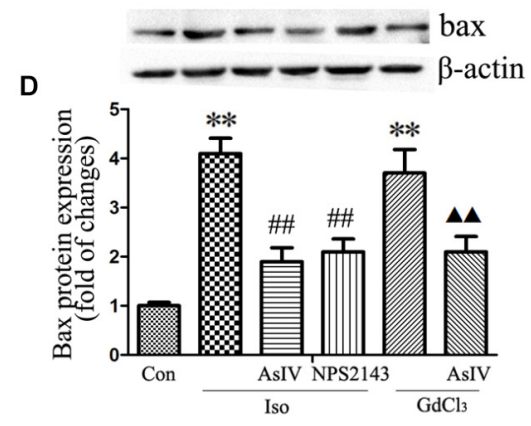

E

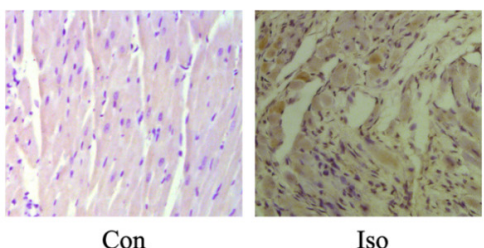

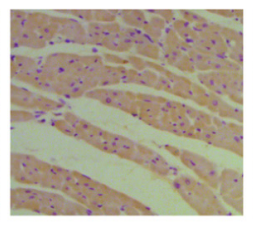

Iso+AsIV

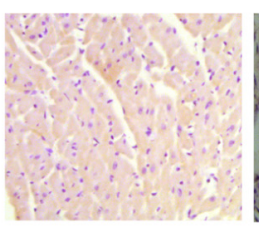

Iso+NPS2143

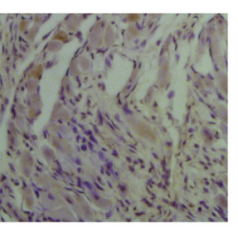

$\mathrm{GdCl}_{3}$

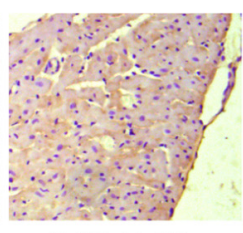

$\mathrm{GdCl}_{3}+\mathrm{AsIV}$
F

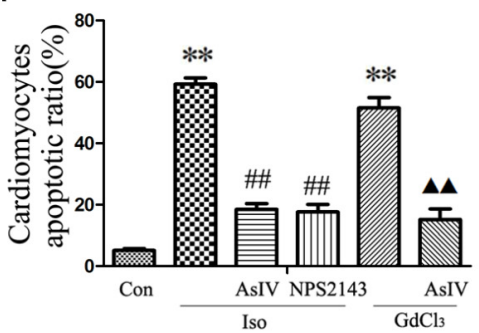

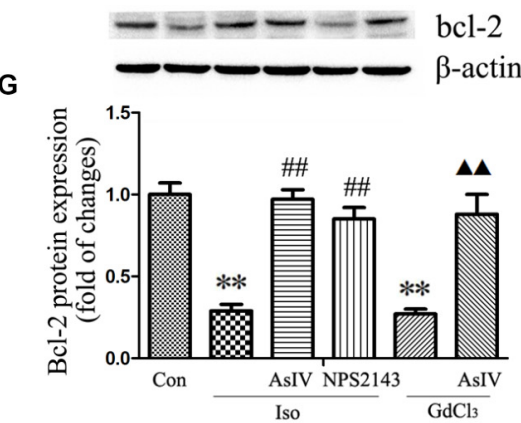
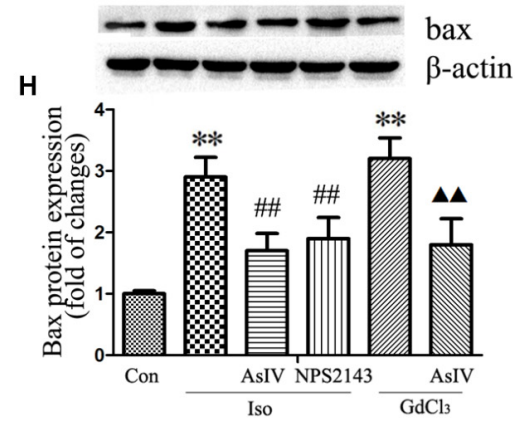

FIGURE 3 | AsIV inhibited apoptosis in hypertrophied heart tissue and cells. (A,B) The level of apoptosis in H9C2 cells was examined using Annexin-V/PI staining and flow cytometry. The upper right area and the lower right area were regarded as apoptotic areas. (C,D) Bax and Bcl-2 protein expression assayed by western blot analysis in $\mathrm{H9C2}$ cells. (E,F) Myocardial apoptosis assayed by TUNEL staining. TUNEL-positive cells were manifested as a marked appearance of dark brown apoptotic cell nuclei. (G,H) Bax and bcl-2 protein expressions assayed by western blot in myocardial tissue. The data are expressed as the means \pm SEM, $n=4$. ${ }^{* *} P<0.01$ vs. the Con group; ${ }^{\#} P<0.01$ vs. the Iso group; and $\mathbf{\Delta} P<0.05, \boldsymbol{\Delta} \mathbf{\Delta} P<0.01$ vs. the $\mathrm{GdCl}_{3}$ group.

to the aggregation of accumulated JC-1, while in apoptotic and dead cells, the dye remains in its monomeric form, and the mitochondria appear green. Therefore, a decreased ratio of red fluorescence to green fluorescence indicates MMP dissipation. The results showed that Iso- or $\mathrm{GdCl}_{3}$ induced decreases in MMP (Figures $\mathbf{4 A}, \mathbf{B}$ ) and mitochondrial cytochrome $\mathrm{c}$ content (Figures $4 \mathrm{C}-\mathrm{E}$ ) were ameliorated by AsIV treatment. In addition, electron microscopy showed that pretreatment with AsIV ameliorated Iso- or $\mathrm{GdCl}_{3}$ induced mitochondrial lesions including mitochondria swelling, cristae breakage, and membrane fusion in cardiac tissue (Figure 4F).

\section{AsIV Decreased CaSR Expression in Heart Tissue and Cells}

Calcium sensing receptor (CaSR) activation contributes to the cardiac injury induced by Iso. To further explore the mechanism underlying the attenuation of cardiac injury by AsIV, the present study investigated the effect of AsIV on 


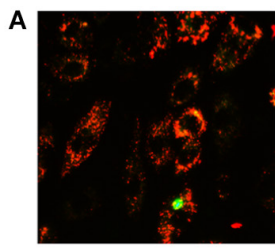

Con

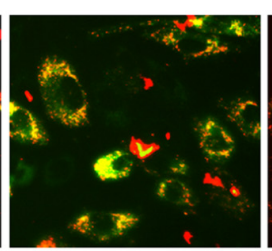

Iso

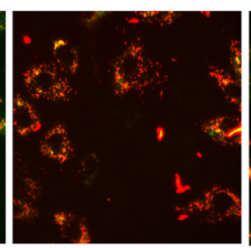

Iso+AsIV

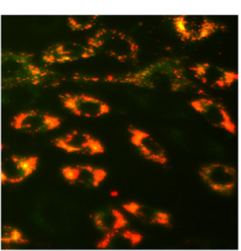

Iso+NPS2143

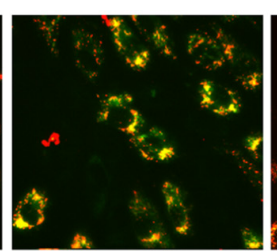

$\mathrm{GdCl}_{3}$

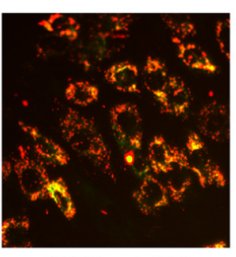

$\mathrm{GdCl}_{3}+\mathrm{AsIV}$

C

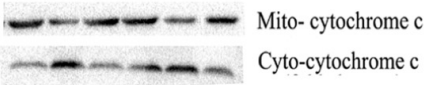

B

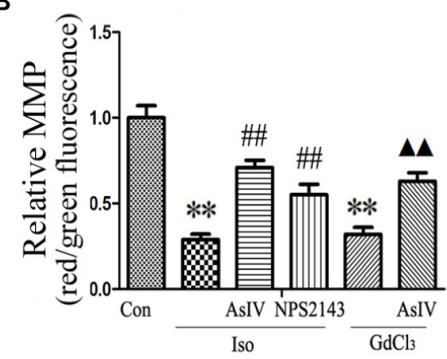

$\mathbf{F}$

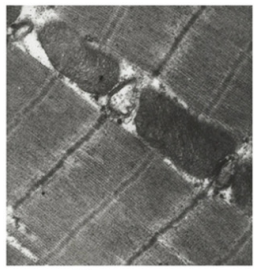

Con

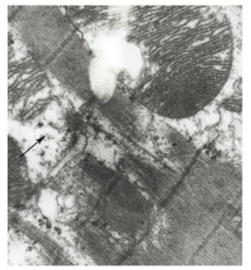

Iso
D
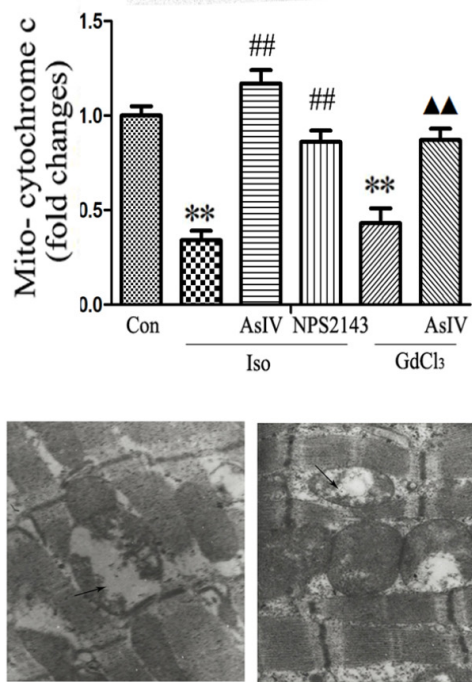

Iso+AsIV

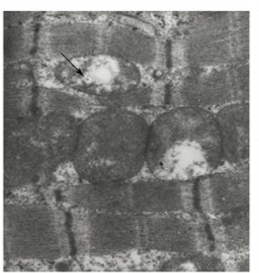

Iso+NPS 2143
$\mathrm{E}$

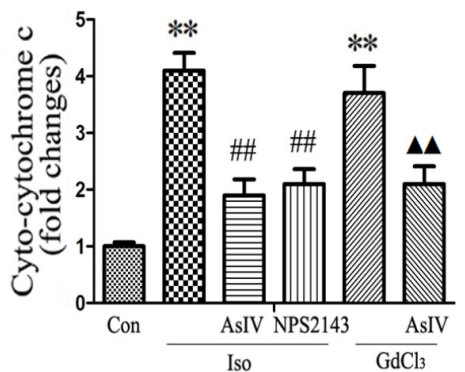

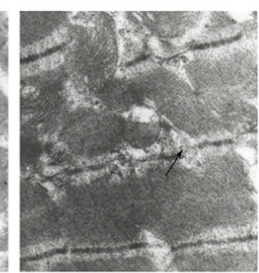

$\mathrm{GdCl}_{3}+\mathrm{AsIV}$

FIGURE 4 | AsIV ameliorated mitochondrial dysfunction in heart tissue and H9C2 cells. (A) H9C2 cells were stained with JC-1, and mitochondrial membrane potential was assessed by fluorescence microscopy. (B) A decreased ratio of red fluorescence to green fluorescence indicates MMP dissipation. (C-E) Mitochondrial and cytoplasmic cytochrome c protein expression assayed by western blot in H9C2 cells. (F) EM was used to detect ultrastructural changes in the left ventricular tissue after the different treatments (magnification $\times 15,000$ ). The arrows indicate the regions of mitochondrial swelling, cristae breakage, and membrane fusion. The data are expressed as the means $\pm \mathrm{SEM}, n=4$. ${ }^{* *} P<0.01$ vs. the Con group; ${ }^{\#} P<0.01$ vs. the Iso group; and $\boldsymbol{\Delta} P<0.05, \boldsymbol{\Delta} \mathbf{\Delta}<0.01$ vs. the GdCl 3 group.

CaSR expression in heart tissue and $\mathrm{H} 9 \mathrm{C} 2$ cell. The results of immunofluorescence analysis in $\mathrm{H} 9 \mathrm{C} 2$ cells (Figure 5A) and western blot analysis in myocardial tissue (Figures 5B,C) showed that the combination of Iso with AsIV significantly suppressed CaSR expression compared with the expression after Iso alone, an effect similar to that of NPS2143. In addition, AsIV suppressed the CaSR expression induced by $\mathrm{GdCl}_{3}$. The results suggested that AsIV exerted a protective effect via inhibiting CaSR activation.

\section{AsIV Regulated $\left[\mathrm{Ca}^{2+}\right]_{\mathrm{i}}$ Induced by Iso and $\mathrm{GdCl}_{3}$}

Activation of CaSR increases $\left[\mathrm{Ca}^{2+}\right]_{i}$ through the PLC-IP3 pathway. To determine the effects of AsIV on increases in $\left[\mathrm{Ca}^{2+}\right]_{\mathrm{i}}$ induced by Iso and CaSR activation, the cells were stained with Fluo-4/AM, and $20 \mu \mathrm{M}$ of 2 -APB (an IP3R inhibitor) was used. The results showed that both Iso and $\mathrm{GdCl}_{3}$ increased $\left[\mathrm{Ca}^{2+}\right]_{i}$, an effect that was inhibited by pretreatment with AsIV. Interestingly, 2-APB completely prevented the $\mathrm{GdCl}_{3}$ induced enhancement of $\left[\mathrm{Ca}^{2+}\right]_{\mathrm{i}}$, but moderately prevented the Iso-induced enhancement of $\left[\mathrm{Ca}^{2+}\right]_{i}$, indicating that the increased $\left[\mathrm{Ca}^{2+}\right]_{\mathrm{i}}$ induced by Iso may be partly due to CaSR activation (Figures 6A,B).

The activity of the calcium pump SERCA2a is considerably reduced in the hypertrophic heart, and changes in SERCA2a activity during hypertrophy contributes substantially to $\mathrm{Ca}^{2+}$ overload. The present study showed the regulatory effect of AsIV on $\left[\mathrm{Ca}^{2+}\right]_{\mathrm{i}}$ and CaSR expression, so we investigated whether AsIV could potentially affect heart SERCA2a and IP3R activity in myocardial tissues. Compared with the expression in the control group, SERCA2a protein expression decreased and IP3R expression increased in the Iso and $\mathrm{GdCl}_{3}$ groups, and these changes were prevented by AsIV treatment (Figures 6C-E).

\section{AsIV Downregulated the CaMKII and CaN Pathways in Heart Tissue and Cells}

Both hypertrophy and apoptosis are regulated by $\mathrm{Ca}^{2+}$ and by complex interactions in intracellular signaling pathways. In the present study, we determined the expression of CaMKII (Figures 7A,C,F,H), CaN (Figures 7A,D,F,I), and GATA-4 (Figures 7A,E,F,J) and the nuclear translocation of NFAT-3 (Figures 7A,B,F,G), all of which are implicated in 
A
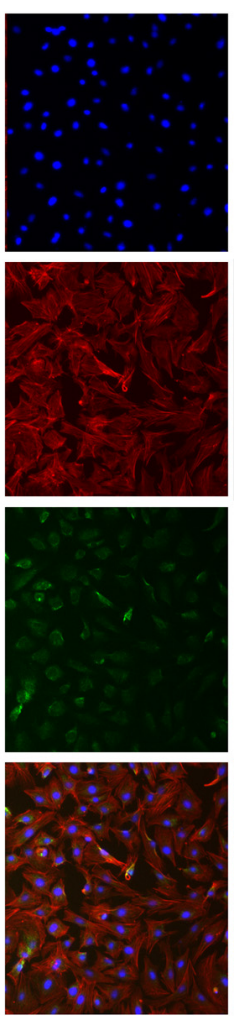

Con
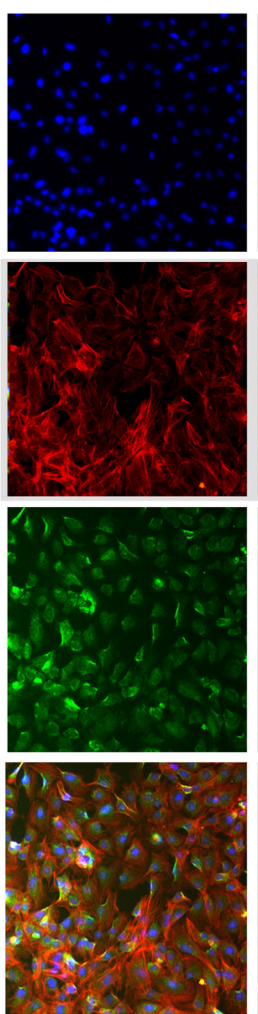

Iso
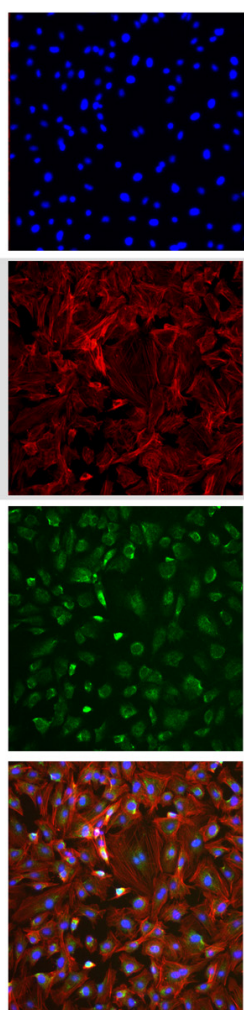

Iso+AsIV
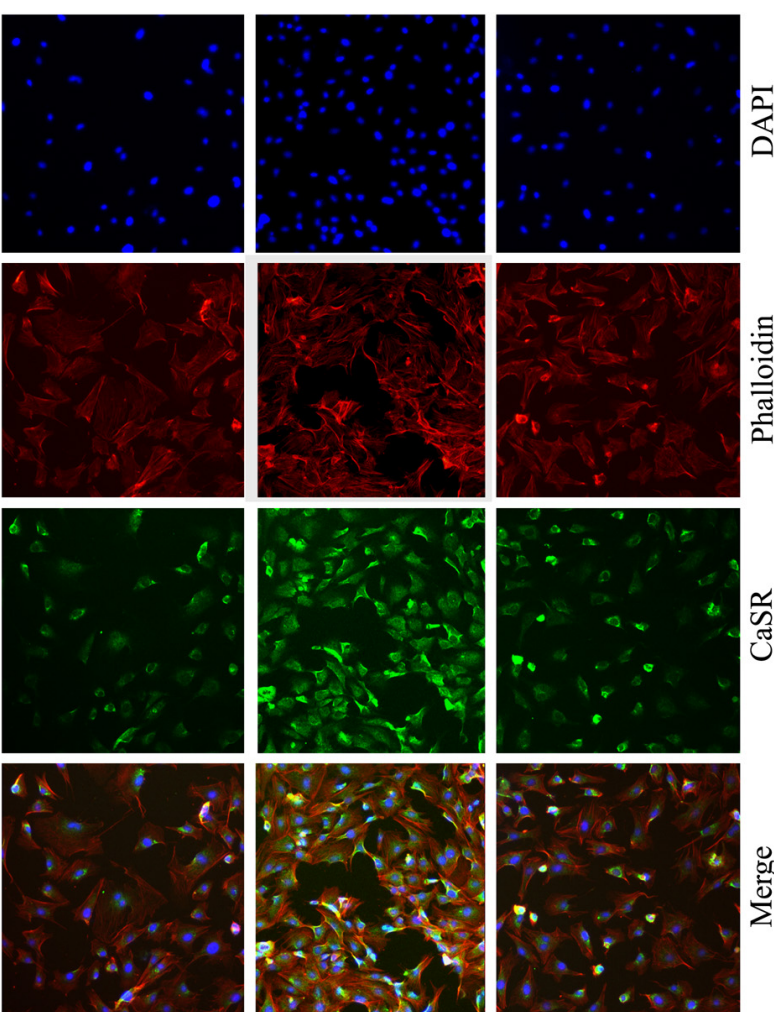

Iso+NPS2143
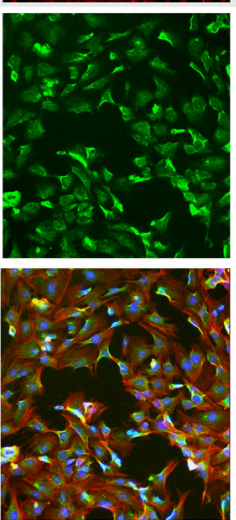

$\mathrm{GdCl}_{3}$
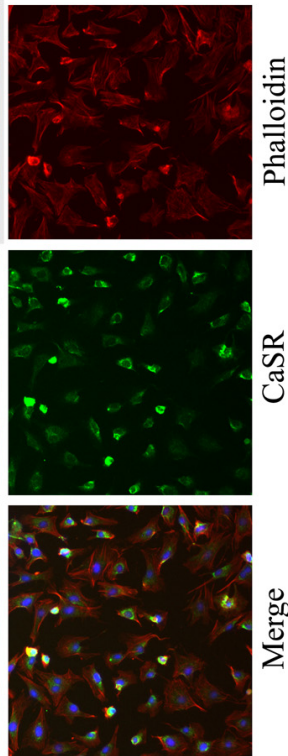

$\mathrm{GdCl}_{3}+\mathrm{AsIV}$
B

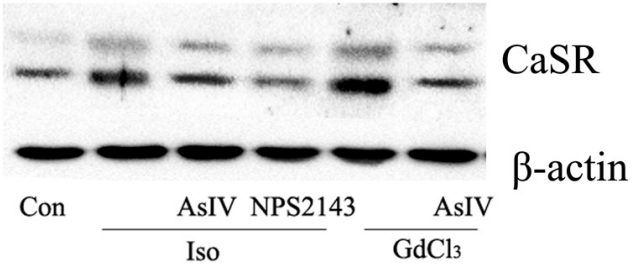

C

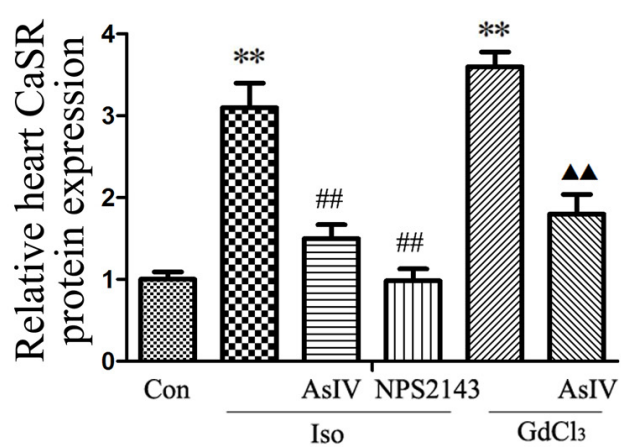

FIGURE 5 | AsIV decreased CaSR expression in heart tissue and cells. (A) The H9C2 cells were incubated with a CaSR antibody overnight at $4^{\circ} \mathrm{C}$, incubated with fluorescein isothiocyanate (FITC)-conjugated goat anti-rabbit secondary antibody, and then treated with rhodamine-labeled phalloidin and DAPI. The cells were examined and photographed using a fluorescence microscope. (B,C) CaSR protein expression as assayed by western blot in myocardial tissue. The data are expressed as the means $\pm \mathrm{SEM}, n=4$. ${ }^{* *} P<0.01$ vs. the Con group; ${ }^{*} P<0.01$ vs. the Iso group; and $\mathbf{\Delta} P<0.05, \boldsymbol{\Delta} \mathbf{\Delta}<0.01$ vs. the GdCl 3 group.

the development of cardiac hypertrophy and apoptosis and are mediated by $\left[\mathrm{Ca}^{2+}\right]_{\mathrm{i}}$. Our results showed that pretreatment with AsIV significantly inhibited the Iso-induced activation of CaMKII, CaN, and GATA-4 and nuclear translocation of NFAT-3, an effect similar to that of NPS2143. Furthermore, CaSR activation with $\mathrm{GdCl}_{3}$ also activated CaMKII, CaN, and GATA-4 and promoted nuclear translocation of NFAT-3, effects that were inhibited by AsIV. These results suggested that the upregulation of CaSR induced by Iso increased $\left[\mathrm{Ca}^{2+}\right]_{i}$, and activated the CaMKII and CaN pathways, subsequently inducing cardiac apoptosis and hypertrophy. AsIV downregulated CaSR expression, inhibited increases in $\left[\mathrm{Ca}^{2+}\right]_{\mathrm{i}}$, inhibited the CaMKII and $\mathrm{CaN}$ pathways, and subsequently alleviated the cardiac injury induced by Iso.

\section{Regulatory Effect of CaSR on CaMKII and CaN Pathways Induced by Iso}

Finally, to confirm the regulatory effect of CaSR on the CaMKII and $\mathrm{CaN}$ pathways induced by Iso, we upregulated CaSR expression through lentivirus infection. The results showed that 
A

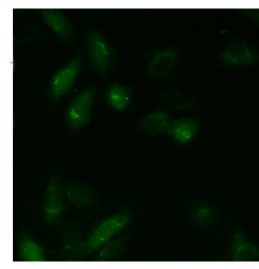

Con

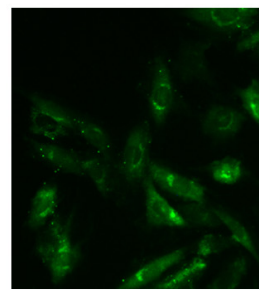

$\mathrm{GdCl}_{3}+\mathrm{AsIV}$

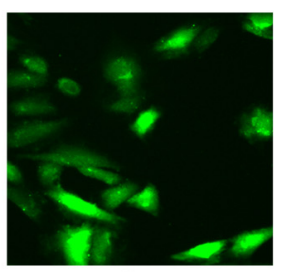

Iso

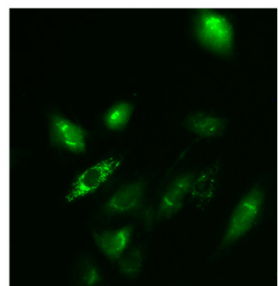

$\mathrm{GdCl3}+2-\mathrm{APB}$

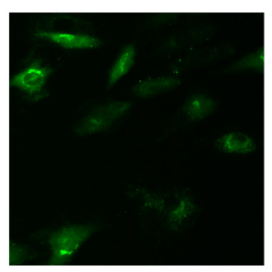

Iso+AsIV

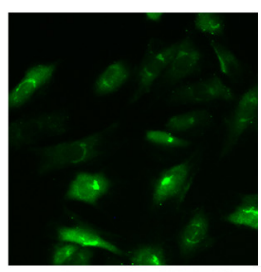

Iso+NPS2143

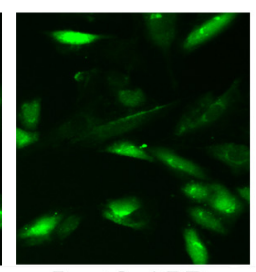

Iso+2-APB

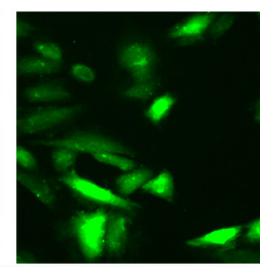

$\mathrm{GdCl}_{3}$

B

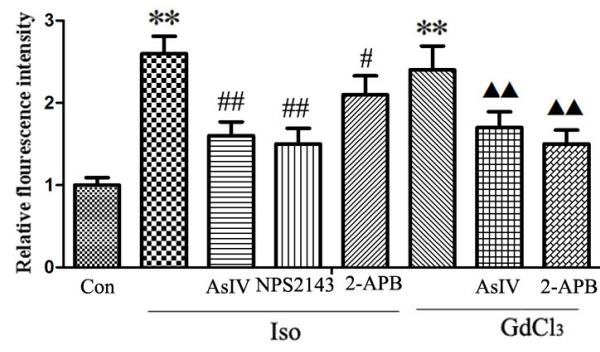

C

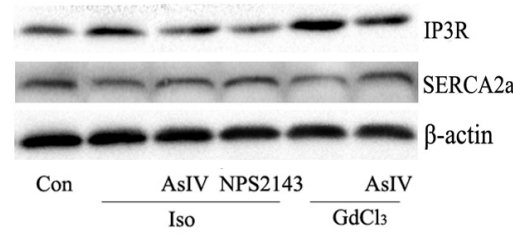

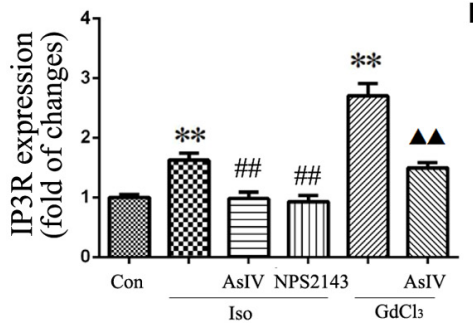

E

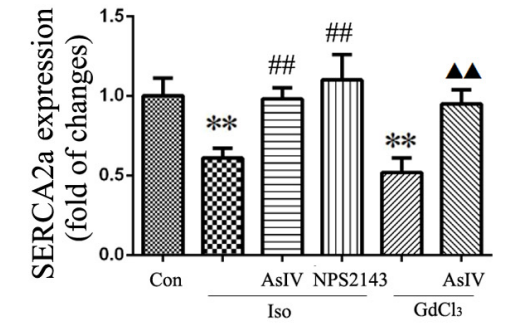

FIGURE 6 | AsIV regulated $\left[\mathrm{Ca}^{2+}\right]_{i}$ induced by Iso and $\mathrm{GdCl}_{3}$. (A) Changes in the intensity of fluorescence of $\left[\mathrm{Ca}^{2+}\right]_{i}$ were recorded with a laser scanning confocal microscope under different treatment conditions. (B) The bars represent the fluorescence relative to that of the controls. (C-E) IP3R and SERCA2a protein expression assayed by western blot analysis in myocardial tissue. Data are expressed as mean \pm SEM, $n=4$. ${ }^{* *} P<0.01$ vs. Con group, ${ }^{*} P<0.01$ vs. Iso group, and $\boldsymbol{\Delta} P<0.05, \Delta \mathrm{\Delta} P<0.01$ vs. $\mathrm{GdCl}_{3}$ group.

CaSR overexpression increased H9C2 cell size (Figures $\mathbf{8 A}, \mathbf{B}$ ), apoptotic ratio (Figures $\mathbf{8 E}, \mathbf{F}$ ), and $\left[\mathrm{Ca}^{2+}\right]_{\mathrm{i}}$ (Figures $\mathbf{9 A , B}$ ), decreased H9C2 MMP (Figures 8C,D), and upregulated the CaMKII and CaN pathways (Figures 9C-G). Furthermore, the effects of CaSR activation on cardiac hypertrophy, apoptosis and the CaMKII and CaN pathways were enhanced by Iso administration. Combined with the abovementioned results, we demonstrated that the activation of $\mathrm{Ca}^{2+}$-dependent CaMKII and $\mathrm{CaN}$ pathways is involved in CaSR-induced cardiac hypertrophy and apoptosis.

\section{DISCUSSION}

Apoptosis and hypertrophy of cardiomyocytes are the primary causes of heart failure and are known to be regulated by complex interactions in the intracellular signaling network. Through extensive computer simulations with various parameter distributions, it has been revealed that $\mathrm{Ca}^{2+}$, CaMKII, and NFAT are consistently found in all the perturbation analyses for cardiac hypertrophy and apoptosis (Liu Y.T. et al., 2016). Activation of CaSR has been reported to be involved in the development of various cardiovascular diseases including cardiac hypertrophy and apoptosis (Schepelmann et al., 2016;
Paquot et al., 2017; Zhang et al., 2018), but the molecular mechanism of the involvement of CaSR activation in heart failure has not yet been clarified and thus needs to be further explored. In the present study, we investigated the role of CaSR activation in cardiac hypertrophy and apoptosis and the relationship between CaSR and $\mathrm{Ca}^{2+}$-dependent CaMKII and $\mathrm{CaN}$ signaling pathways both in vivo and in vitro. The results demonstrated that CaSR activation contributed to cardiac hypertrophy and apoptosis, and the effect of CaSR was mediated by the activation of $\mathrm{Ca}^{2+}$-dependent CaMKII and CaN signaling pathways. What is more, AsIV suppressed CaSR activation, downregulated $\mathrm{Ca}^{2+} / \mathrm{CaMKII} / \mathrm{CaN}$ signaling pathways, and subsequently attenuated cardiac hypertrophy and apoptosis induced by Iso (Figure 10).

We previously reported that AsIV attenuated Iso-induced myocardial injury by inhibiting calpain-1 activation and the TLR4/NF- $\mathrm{B}$ signaling pathway and by correcting energy biosynthesis dysfunction (Yang et al., 2013; Mei et al., 2015; Zhang et al., 2015). The present study confirmed the findings of our previous studies that AsIV treatment attenuates cardiac hypertrophy and apoptosis, as reflected by the (1) decreases in HW/BW, LVW/BW, cardiomyocyte crosssectional diameter, ventricular wall thickness, and TUNELpositive cells in myocardial tissue, and (2) decreases in cell 


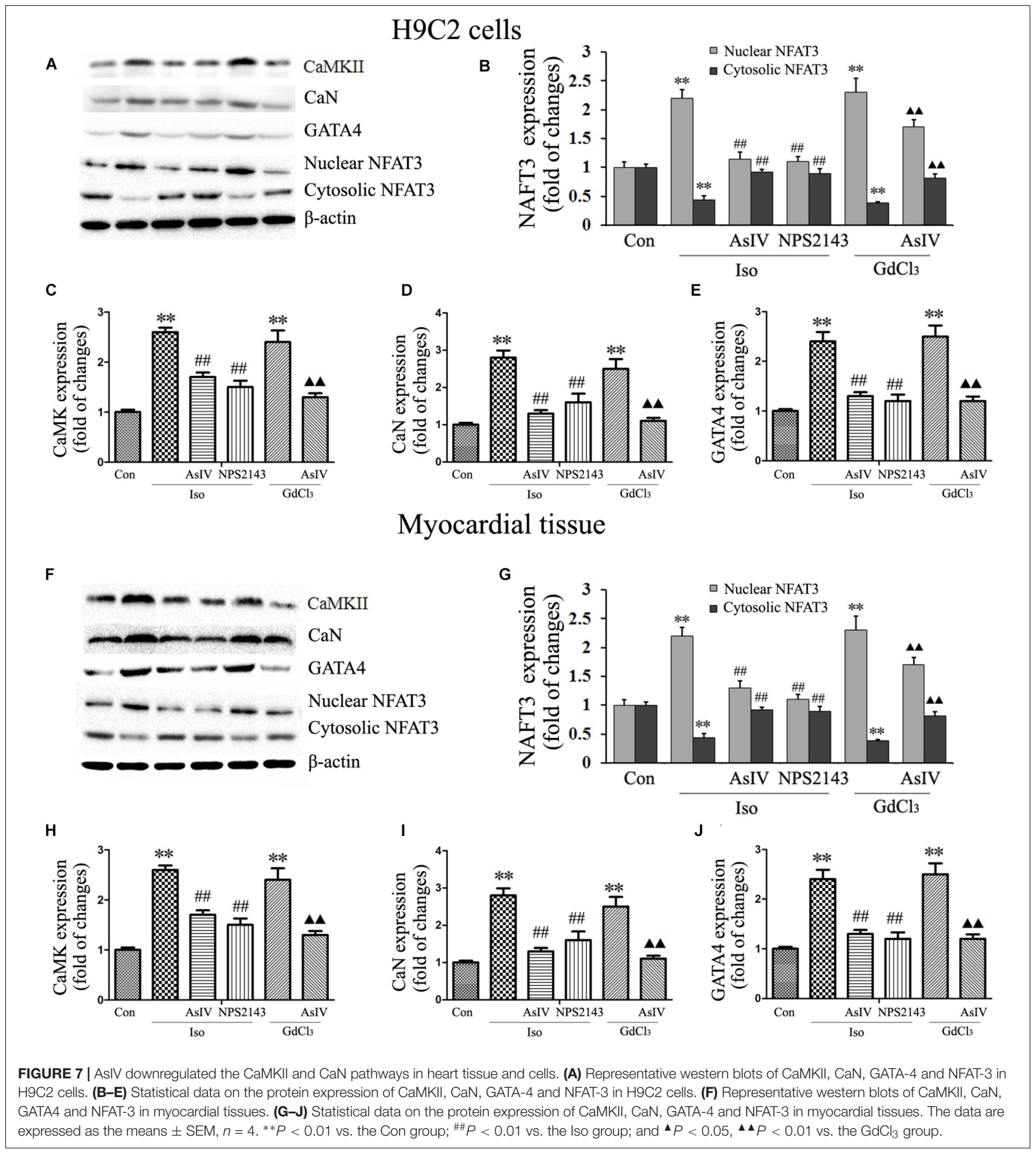

size, ANP and BNP mRNA expression and Annexin-V/PIpositive cells in $\mathrm{H} 9 \mathrm{C} 2$ cells. The novel finding is that the inhibitory action of AsIV was accompanied by downregulations in CaSR expression and $\left[\mathrm{Ca}^{2+}\right]_{\mathrm{i}}$, and the inhibitory effect of AsIV was similar to that of the CaSR antagonist NPS2143. It has been previously reported that CaSR activation with
$\mathrm{GdCl}_{3}$ can induce cardiac hypertrophy and apoptosis ( $\mathrm{Lu}$ et al., 2013). As an agonist of $\mathrm{CaSR}, \mathrm{GdCl}_{3}$ gets involved in cardiac hypertrophy and apoptosis by activating CaSR. Activation of CaSR increases $\left[\mathrm{Ca}^{2+}\right]_{\mathrm{i}}$, then triggers activation of hypertrophic and apoptotic signaling pathways, which are regulated by $\left[\mathrm{Ca}^{2+}\right]_{i}$. AsIV also attenuated $\mathrm{GdCl}_{3}$-induced 
A

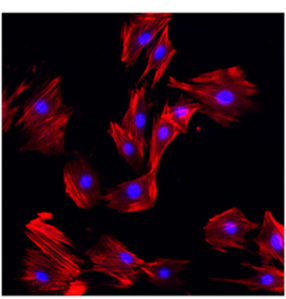

Con

C

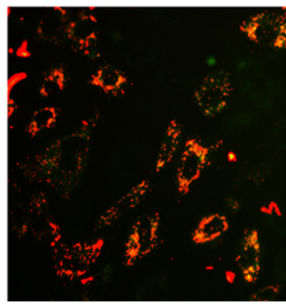

Con

E

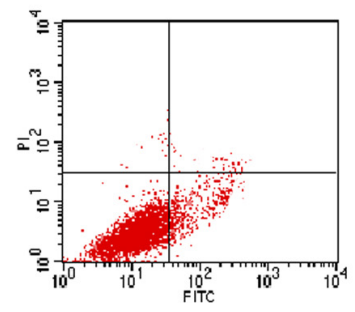

Con

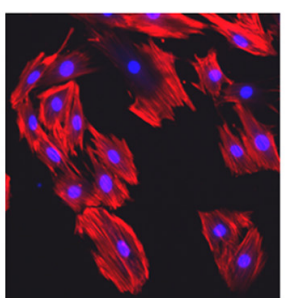

CaSR

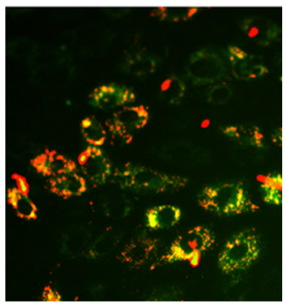

CaSR

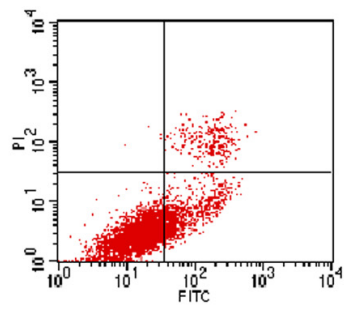

CaSR

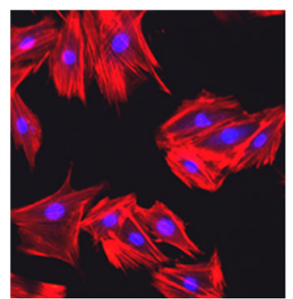

CaSR+Iso

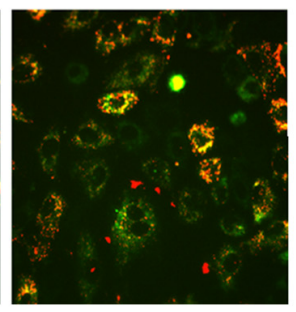

CaSR+Iso

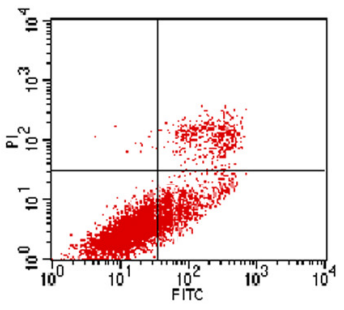

CaSR+Iso
B

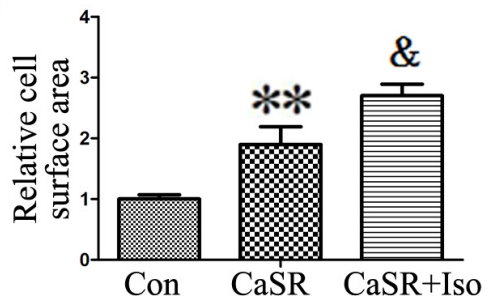

D

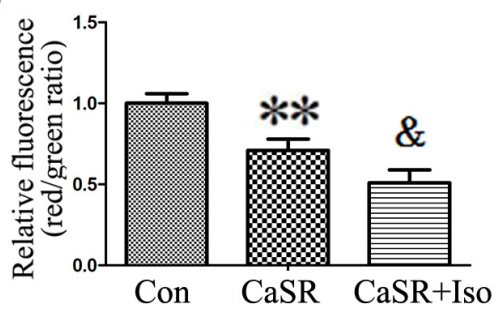

F

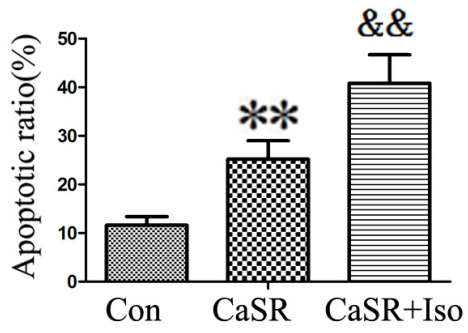

FIGURE 8 | Regulatory effect of CaSR overexpression on cell hypertrophy and apoptosis. (A,B) H9C2 cells were stained with rhodamine-labeled phalloidin and DAPI, and the cell surface area was measured and analyzed with LAS Software (V4.3) (Leica, Germany). The bars represent the cell size relative to that of the controls. (C,D) H9C2 cells were stained with JC-1, and the bars represent the fluorescence relative to that of the controls. (E,F) The level of apoptosis in H9C2 cells was examined using Annexin-V/PI staining and flow cytometry. The data are expressed as the means \pm SEM, $n=4$. ${ }^{* *} P<0.01$ vs. the Con group; and \& $P<0.05$; $\& \& P<0.01$ vs. the shCaSR group.

cardiac hypertrophy and apoptosis through the downregulation of CaSR expression and $\left[\mathrm{Ca}^{2+}\right]_{\mathrm{i}}$. CaSR detects changes in extracellular calcium concentrations $\left(\left[\mathrm{Ca}^{2+}\right]_{\mathrm{o}}\right)$. Increased levels of $\left[\mathrm{Ca}^{2+}\right]_{0}$ induce the binding of $\mathrm{Ca}^{2+}$ to CaSR and activate the G-protein-PLC-IP 3 receptor pathway, which triggers the elevation of $\left[\mathrm{Ca}^{2+}\right]_{i}$, thus initiating numerous effects (Brown and MacLeod, 2001). Under physiological conditions, $\mathrm{Ca}^{2+}$ enters cardiomyocytes through the L-type $\mathrm{Ca}^{2+}$ channel (LTCC) and the $\beta$-adrenergic receptor $(\beta$-AR), causing the release of a large amount of $\mathrm{Ca}^{2+}$ from the Sarcoplasmic Reticulum (SR) through activation of the RyR and leading to contraction. However, under some pathological conditions, such as pulmonary hypertension, myocardial ischemia, and cardiac hypertrophy, CaSR expression is upregulated, leading to $\left[\mathrm{Ca}^{2+}\right]_{\mathrm{i}}$ enhancement and thus aggravating the progression of these diseases (Guo et al., 2012, 2017; Zhang et al., 2016). Upon review of the previous studies, it was found that CaSR contributes to cardiac hypertrophy induced by angiotensin II, Iso, and transverse aortic constriction, and the mechanism may involve $\left[\mathrm{Ca}^{2+}\right]_{i}$, the sarcoplasmic reticulum (ER), the mitochondrial death pathway and autophagy (Wang et al., 2008; Lu et al., 2013; Liu L. et al., 2016). Downregulation of CaSR with Calhex-231 and NPS2143 reduces vascular reactivity via inhibition of voltagegated $\mathrm{Ca}^{2+}$ channels (Greenberg et al., 2016). Consistent with the results of previous studies, the present study demonstrated that CaSR contributed to cardiac hypertrophy and apoptosis induced by Iso but that the mechanism may be related to mitochondrial dysfunction and $\mathrm{Ca}^{2+}$-dependent CaMKII and CaN pathways.

Mitochondrial dysfunction contributes to multiple types of damage, including cardiac hypertrophy and apoptosis. The mitochondrial release of cytochrome $c$ was analyzed to prove the involvement of the mitochondrial apoptotic pathway in heart failure. In our experiment, Iso administration destroyed mitochondrial structure in rats and reduced the MMP and mitochondrial cytochrome $\mathrm{c}$ in $\mathrm{H} 9 \mathrm{C} 2$ cells, indicating mitochondrial dysfunction. Pretreatment with AsIV attenuated cardiac hypertrophy and apoptosis, improved cardiac function, and downregulated CaSR expression while also improving mitochondrial structure and MMP and inhibiting cytochrome c release from the mitochondria. A previous study indicated that CaSR activation caused $\mathrm{Ca}^{2+}$ release from the SR into the mitochondria and induced cardiomyocyte apoptosis and that changes in SERCA2a activity during HF contribute 
A

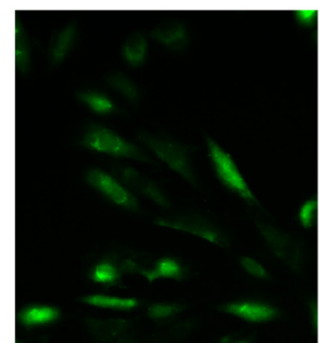

Con

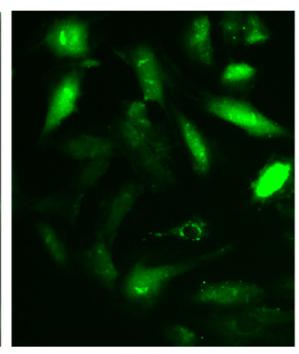

CaSR

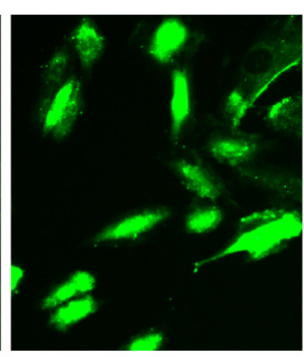

CaSR+Iso
B

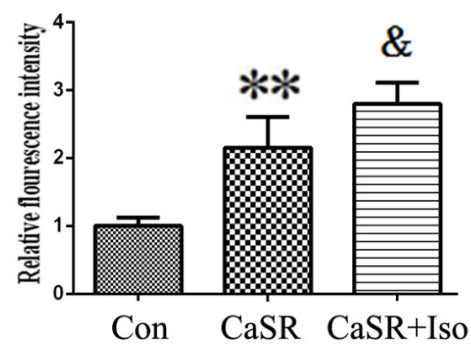

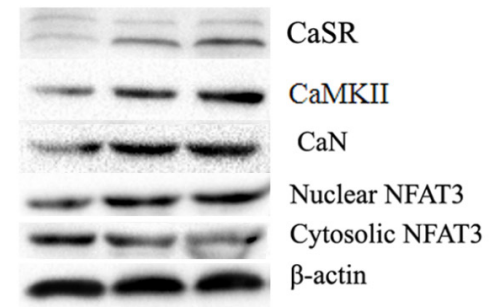

E

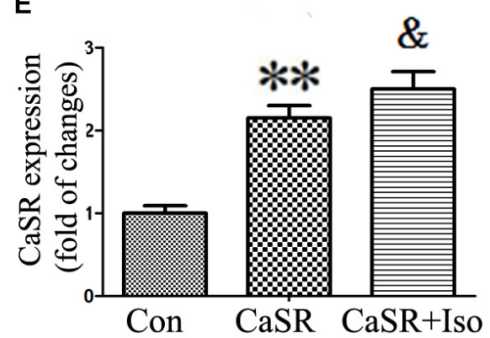

D

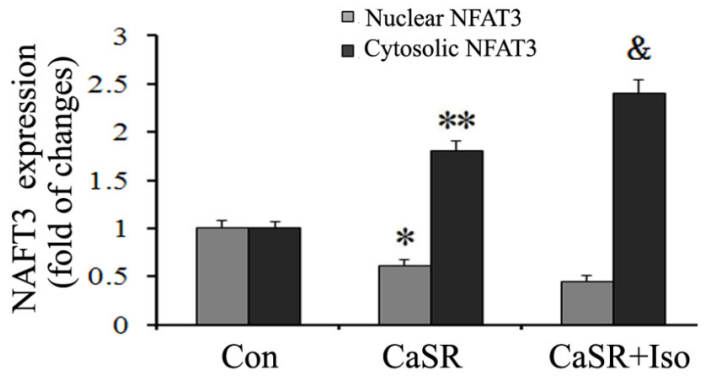

F

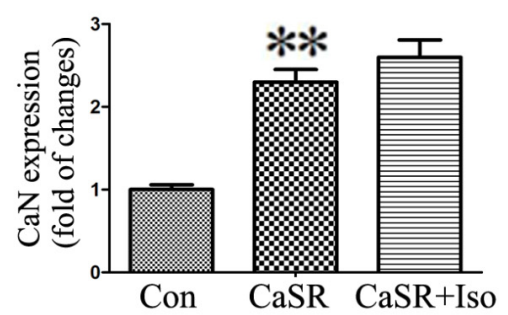

G

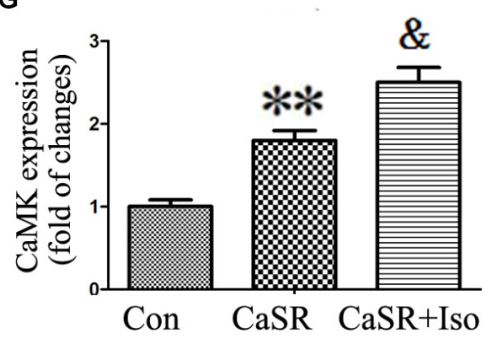

FIGURE 9 | Regulatory effect of CaSR activation on $\left[\mathrm{Ca}^{2+}\right]_{i}$ and the CaMKII and CaN pathways. (A) Changes in the intensity of fluorescence of [Ca $\left.{ }^{2+}\right]_{i}$ were recorded with a laser scanning confocal microscope under different treatment conditions. (B) The bars represent the fluorescence relative to that of the controls. (C-G) CaSR, CaMKII, CaN and NFAT-3 protein expression assayed by western blot in H9C2 cells. The data are expressed as the means \pm SEM, $n=4$. ${ }^{* *}<0.01$ vs. the Con group; \& $P<0.05$ vs. the CaSR group.

substantially to $\mathrm{Ca}^{2+}$ overload and mitochondrial dysfunction (Dong et al., 2017). However, the present study investigated $\left[\mathrm{Ca}^{2+}\right]_{\mathrm{i}}$ using Fluo-4/AM and found that the IP3Rs inhibitor 2-APB completely prevented the $\mathrm{GdCl}_{3}$-induced enhancement of $\left[\mathrm{Ca}^{2+}\right]_{\mathrm{i}}$ but only moderately prevented the Iso-induced enhancement of $\left[\mathrm{Ca}^{2+}\right]_{i}$. Pretreatment with AsIV inhibited the enhancement of $\left[\mathrm{Ca}^{2+}\right]_{i}$ induced by Iso and $\mathrm{GdCl}_{3}$. In addition, SERCA2a protein expression decreased and IP3R expression increased in the Iso and $\mathrm{GdCl}_{3}$ groups, effects that were prevented by AsIV treatment. These results suggested that the increased $\left[\mathrm{Ca}^{2+}\right]_{\mathrm{i}}$ induced by Iso may be partly due to CaSR activation, and inhibition of $\left[\mathrm{Ca}^{2+}\right]_{i}$ contributes to the protective effect of AsIV against Iso-induced cardiac injury.

Increasing evidence has demonstrated that the pathway of cell hypertrophy and apoptosis induced by $\mathrm{CaSR} / \mathrm{Ca}^{2+}$ is one of the major contributors to cardiac injury. To investigate the underlying mechanism, we evaluated the CaMKII and $\mathrm{CaN}$ signaling pathways, both of which were regulated by $\left[\mathrm{Ca}^{2+}\right]_{\mathrm{i}}$. CaMKII is involved in cardiac hypertrophy and apoptosis induced by Iso and testosterone (Bin-Dayel et al., 2016; Duran et al., 2017; Park et al., 2018), and at the epigenetic level, CaMKII inactivates the negative regulator of adverse cardiac remodeling histone deacetylase 4 (HDAC4), leading to transcriptional activation of the myocyte enhancer factor 2 (MEF2), and phosphorylates histone H3 (Backs et al., 2009; Awad et al., 2013). CaN triggers apoptosis and hypertrophy either by dephosphorylating NFAT to activate the subsequent transcription of apoptosis and hypertrophy genes or by dephosphorylating Bad and thereby facilitating the binding of Bad to anti-apoptotic proteins (Lu et al., 2014; Lin et al., 2017). The current study showed that upregulation of CaSR was accompanied by increased CaMKII and CaN expression and NFAT-3 nuclear translocation induced by Iso or $\mathrm{GdCl}_{3}$. NPS2143, an inhibitor of CaSR, not only downregulated CaSR expression but also inhibited CaMKII and CaN expression and NFAT-3 nuclear translocation induced by Iso. Finally, in order to further elucidate the molecular mechanism by which CaSR contributes to cardiac injury induced by Iso, we established a gain-of-function mutation in CaSR by 


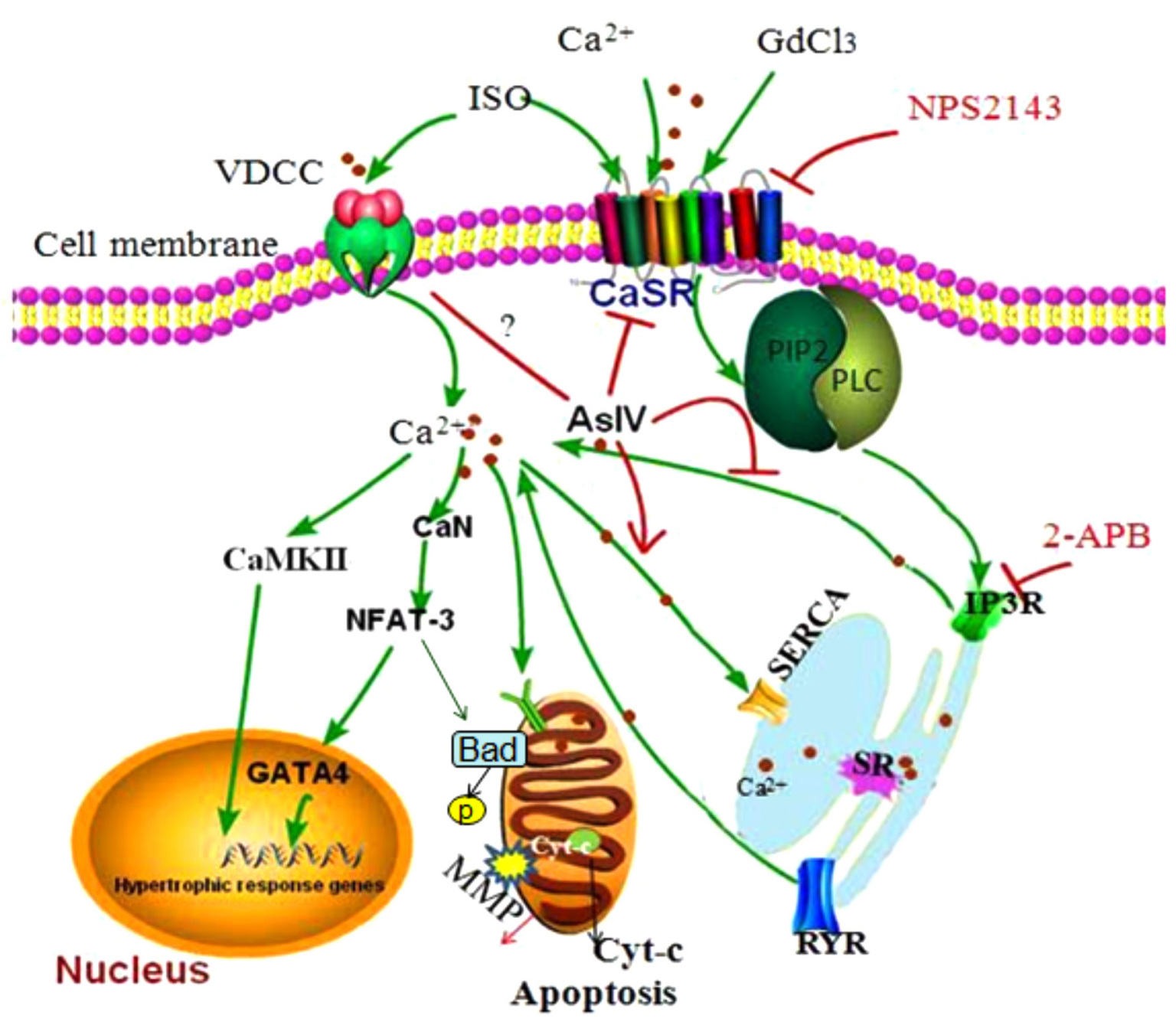

FIGURE 10 | The diagram demonstrating the mechanism of cardioprotection by AsIV via downregulation of CaSR. The stimulation of cardiomyocytes by excessive extracellular $\mathrm{Ca}^{2+}$, Iso and $\mathrm{GdCl}_{3}$ induces overload of $\left[\mathrm{Ca}^{2+}\right]_{i}$ through CaSR/IP3R pathway. The increased $\left[\mathrm{Ca}^{2+}\right]_{i}$ leads to activation of CaMKII and CaN signaling pathway, resulting in cardiac hypertrophy and apoptosis. AsIV inhibits cardiac hypertrophy and apoptosis through downregulation of CaSR and IP3R expression and activation of SERCA 2 a.

lentivirus infection. As we expected, CaSR activation by lentivirus infection resulted in CaSR upregulation, $\left[\mathrm{Ca}^{2+}\right]_{\mathrm{i}}$ overload, and CaMKII and CaN pathway activation as well as cardiomyocyte hypertrophy and apoptosis. In addition, all of the aforementioned effects were enhanced by Iso, suggesting that regulation of $\left[\mathrm{Ca}^{2+}\right]_{\mathrm{i}}$ and $\mathrm{Ca}^{2+}$-dependent CaMKII and $\mathrm{CaN}$ pathways is the molecular mechanism by which CaSR contributes to cardiac injury induced by Iso. Furthermore, pretreatment with AsIV had an effect similar to that of NPS2143 and inhibited the activation of the CaMKII and CaN pathways, indicating that the protective effect of AsIV against cardiac injury may be partly mediated via the inhibition of CaSR, $\left[\mathrm{Ca}^{2+}\right]_{\mathrm{i}}$ elevation and $\mathrm{Ca}^{2+}$-dependent pathways. However, the present study has limitation. Although H9C2 cell line is commonly used as in vitro model of cardiomyocyte biology, it is a tumor line from atrial rat tumors with the disadvantages of not beating and not postmitotic like usually adult cardiomyocytes.
So we will investigate the cardioprotective effect of AsIV using adult cardiomyocytes in further study. This study is of clinical significance for providing the evidence that AsIV might be potentially used in the prevention and treatment of hypertrophic cardiomyopathy.

\section{CONCLUSION}

The present study demonstrated, for the first time, that CaSR contributes to cardiac hypertrophy and apoptosis by increasing $\left[\mathrm{Ca}^{2+}\right]_{\mathrm{i}}$ and activating $\mathrm{Ca}^{2+}$-dependent CaMKII and $\mathrm{CaN}$ pathways. The cardioprotective effect of AsIV on Iso-induced cardiac injury may be partly mediated via the inhibition of CaSR, $\left[\mathrm{Ca}^{2+}\right]_{\mathrm{i}}$ elevation and, $\mathrm{Ca}^{2+}$-dependent pathways. This study provides a new perspective for developing AsIV as a therapeutic candidate for cardiovascular disease. 


\section{AUTHOR CONTRIBUTIONS}

HW and ML conceived and designed the experiments. ML, BL, and $\mathrm{XH}$ performed the experiments. ML and $\mathrm{ZZ}$ analyzed the data. ML and FT wrote or modified the paper. All authors contributed to and approved the final draft of the manuscript.

\section{REFERENCES}

Awad, S., Kunhi, M., Little, G. H., Bai, Y., An, W., Bers, D., et al. (2013). Nuclear CaMKII enhances histone $\mathrm{H} 3$ phosphorylation and remodels chromatin during cardiac hypertrophy. Nucleic Acids Res. 41, 7656-7672. doi: 10.1093/nar/gkt500

Backs, J., Backs, T., Neef, S., Kreusser, M. M., Lehmann, L. H., Patrick, D. M., et al. (2009). The delta isoform of CaM kinase II is required for pathological cardiac hypertrophy and remodeling after pressure overload. Proc. Natl. Acad. Sci. U.S.A. 106, 2342-2347. doi: 10.1073/pnas.0813013106

Bin-Dayel, A. F., Abdel Baky, N. A., Fadda, L. M., Mohammad, R. A., and AlMohanna, F. (2016). Effect of aliskiren and carvedilol on expression of $\mathrm{Ca}(2$ + )/calmodulin-dependent protein kinase II delta-subunit isoforms in cardiac hypertrophy rat model. Toxicol. Mech. Methods 26, 122-131. doi: 10.3109/ 15376516.2015.1128035

Brown, E. M., and MacLeod, R. J. (2001). Extracellular calcium sensing and extracellular calcium signaling. Physiol. Rev. 81, 239-297. doi: 10.1152/physrev. 2001.81.1.239

Dewenter, M., Neef, S., Vettel, C., Lammle, S., Beushausen, C., Zelarayan, L. C., et al. (2017). Calcium/calmodulin-dependent protein kinase II activity persists during chronic beta-adrenoceptor blockade in experimental and human heart failure. Circ. Heart Fail. 10:e003840. doi: 10.1161/CIRCHEARTFAILURE.117. 003840

Dong, Z., Zhao, P., Xu, M., Zhang, C., Guo, W., Chen, H., et al. (2017). Astragaloside IV alleviates heart failure via activating PPARalpha to switch glycolysis to fatty acid beta-oxidation. Sci. Rep. 7:2691. doi: 10.1038/s41598017-02360-5

Duran, J., Lagos, D., Pavez, M., Troncoso, M. F., Ramos, S., Barrientos, G., et al. (2017). $\mathrm{Ca}(2+) /$ calmodulin-dependent protein kinase II and androgen signaling pathways modulate MEF2 activity in testosterone-induced cardiac myocyte hypertrophy. Front. Pharmacol. 8:604. doi: 10.3389/fphar.2017.00604

Greenberg, H. Z. E., Jahan, K. S., Shi, J., Vanessa Ho, W. S., and Albert, A. P. (2016). The calcilytics Calhex-231 and NPS 2143 and the calcimimetic calindol reduce vascular reactivity via inhibition of voltage-gated $\mathrm{Ca}(2+)$ channels. Eur. J. Pharmacol. 791, 659-668. doi: 10.1016/j.ejphar.2016.10.008

Gui, J., Chen, R., Xu, W., and Xiong, S. (2015). Remission of CVB3-induced myocarditis with Astragaloside IV treatment requires A20 (TNFAIP3) upregulation. J. Cell Mol. Med. 19, 850-864. doi: 10.1111/jcmm.12459

Guo, H., Cao, A., Chu, S., Wang, Y., Zang, Y., Mao, X., et al. (2016) Astragaloside IV attenuates podocyte apoptosis mediated by endoplasmic reticulum stress through upregulating sarco/endoplasmic reticulum $\mathrm{Ca}(2+)$ ATPase 2 expression in diabetic nephropathy. Front. Pharmacol. 7:500. doi: 10.3389/fphar.2016.00500

Guo, J., Li, H. Z., Wang, L. C., Zhang, W. H., Li, G. W., Xing, W. J., et al. (2012). Increased expression of calcium-sensing receptors in atherosclerosis confers hypersensitivity to acute myocardial infarction in rats. Mol. Cell. Biochem. 366, 345-354. doi: 10.1007/s11010-012-1312-0

Guo, Q., Xu, H., Yang, X., Zhao, D., Liu, S., Sun, X., et al. (2017). Notch activation of $\mathrm{Ca}(2+)$-sensing receptor mediates hypoxia-induced pulmonary hypertension. Hypertens. Res. 40, 117-129. doi: 10.1038/hr.2016.118

Hong, S., Zhang, X., Zhang, X., Liu, W., Fu, Y., Liu, Y., et al. (2017). Role of the calcium sensing receptor in cardiomyocyte apoptosis via mitochondrial dynamics in compensatory hypertrophied myocardium of spontaneously hypertensive rat. Biochem. Biophys. Res. Commun. 487, 728-733. doi: 10.1016/j. bbrc.2017.04.126

Jiang, C. M., Han, L. P., Li, H. Z., Qu, Y. B., Zhang, Z. R., Wang, R., et al. (2008). Calcium-sensing receptors induce apoptosis in cultured neonatal rat ventricular cardiomyocytes during simulated ischemia/reperfusion. Cell Biol. Int. 32, 792-800. doi: 10.1016/j.cellbi.2008.03.009

\section{FUNDING}

This work was supported by National Natural Science Foundation of China (81673632, 81703739, and 81870329), Natural Science Foundation of Liaoning Province (Nos. 201602317 and 20180530069).

Jiang, X. G., Sun, K., Liu, Y. Y., Yan, L., Wang, M. X., Fan, J. Y., et al. (2017). Astragaloside IV ameliorates 2,4,6-trinitrobenzene sulfonic acid (TNBS)induced colitis implicating regulation of energy metabolism. Sci. Rep. 7:41832. doi: $10.1038 /$ srep 41832

Khalilimeybodi, A., Daneshmehr, A., and Sharif-Kashani, B. (2017). Investigating beta-adrenergic-induced cardiac hypertrophy through computational approach: classical and non-classical pathways. J. Physiol. Sci. 68, 503-520. doi: 10.1007/s12576-017-0557-5

Kim, T. Y., Terentyeva, R., Roder, K. H., Li, W., Liu, M., Greener, I., et al. (2017). SK channel enhancers attenuate Ca2 +-dependent arrhythmia in hypertrophic hearts by regulating mito-ROS-dependent oxidation and activity of RyR. Cardiovasc. Res. 113, 343-353. doi: 10.1093/cvr/cvx005

Li, C., Yang, F., Liu, F., Li, D., and Yang, T. (2018). NRF2/HO-1 activation via ERK pathway involved in the anti-neuroinflammatory effect of Astragaloside IV in LPS induced microglial cells. Neurosci. Lett. 666, 104-110. doi: 10.1016/j.neulet. 2017.12.039

Li, H. X., Kong, F. J., Bai, S. Z., He, W., Xing, W. J., Xi, Y. H., et al. (2012). Involvement of calcium-sensing receptor in oxLDL-induced MMP-2 production in vascular smooth muscle cells via PI3K/Akt pathway. Mol. Cell. Biochem. 362, 115-122. doi: 10.1007/s11010-011-1133-6

Li, X. M., Ma, Y. T., Yang, Y. N., Liu, F., Chen, B. D., Han, W., et al. (2009). Downregulation of survival signalling pathways and increased apoptosis in the transition of pressure overload-induced cardiac hypertrophy to heart failure. Clin. Exp. Pharmacol. Physiol. 36, 1054-1061. doi: 10.1111/j.1440-1681.2009. 05243.x

Li, X. Z., Ding, Y. Z., Wu, H. F., Bian, Z. P., Xu, J. D., Gu, C. R., et al. (2017). Astragaloside IV prevents cardiac remodeling in the apolipoprotein E-deficient mice by regulating cardiac homeostasis and oxidative stress. Cell. Physiol. Biochem. 44, 2422-2438. doi: 10.1159/000486166

Lin, K. H., Kuo, W. W., Shibu, M. A., Day, C. H., Hsieh, Y. L., Chung, L. C., et al. (2017). E2/ER beta enhances calcineurin protein degradation and PI3K/Akt/MDM2 signal transduction to inhibit ISO-induced myocardial cell apoptosis. Int. J. Mol. Sci. 18:892. doi: 10.3390/ijms18040892

Liu, L., Wang, C., Lin, Y., Xi, Y., Li, H., Shi, S., et al. (2016). Suppression of calciumsensing receptor ameliorates cardiac hypertrophy through inhibition of autophagy. Mol. Med. Rep. 14, 111-120. doi: 10.3892/mmr.2016.5279

Liu, Y. T., Zhou, C., Jia, H. M., Chang, X., and Zou, Z. M. (2016). Standardized Chinese formula Xin-Ke-Shu inhibits the myocardium $\mathrm{Ca}(2+)$ overloading and metabolic alternations in isoproterenol-induced myocardial infarction rats. Sci. Rep. 6:30208. doi: 10.1038/srep30208

Lu, F., Tian, Z., Zhang, W., Zhao, Y., Bai, S., Ren, H., et al. (2010). Calcium-sensing receptors induce apoptosis in rat cardiomyocytes via the endo(sarco)plasmic reticulum pathway during hypoxia/reoxygenation. Basic Clin. Pharmacol. Toxicol. 106, 396-405. doi: 10.1111/j.1742-7843.2009.00502.x

Lu, F. H., Fu, S. B., Leng, X., Zhang, X., Dong, S., Zhao, Y. J., et al. (2013). Role of the calcium-sensing receptor in cardiomyocyte apoptosis via the sarcoplasmic reticulum and mitochondrial death pathway in cardiac hypertrophy and heart failure. Cell. Physiol. Biochem. 31, 728-743. doi: 10.1159/000350091

Lu, M., Wang, H., Wang, J., Zhang, J., Yang, J., Liang, L., et al. (2014). Astragaloside IV protects against cardiac hypertrophy via inhibiting the $\mathrm{Ca} 2+/ \mathrm{CaN}$ signaling pathway. Planta Med. 80, 63-69. doi: 10.1055/s-0033-1360129

Mei, M., Tang, F., Lu, M., He, X., Wang, H., Hou, X., et al. (2015). Astragaloside IV attenuates apoptosis of hypertrophic cardiomyocyte through inhibiting oxidative stress and calpain-1 activation. Environ. Toxicol. Pharmacol. 40, 764-773. doi: 10.1016/j.etap.2015.09.007

Paquot, F., Huart, J., Defraigne, J. O., Krzesinski, J. M., and Jouret, F. (2017) Implications of the calcium-sensing receptor in ischemia/reperfusion. Acta Cardiol. 72, 125-131. doi: 10.1080/00015385.2017.1291136 
Park, S. W., Persaud, S. D., Ogokeh, S., Meyers, T. A., Townsend, D., and Wei, L. N. (2018). CRABP1 protects the heart from isoproterenol-induced acute and chronic remodeling. J. Endocrinol. 236, 151-165. doi: 10.1530/JOE-17-0613

Schepelmann, M., Yarova, P. L., Lopez-Fernandez, I., Davies, T. S., Brennan, S. C., Edwards, P. J., et al. (2016). The vascular Ca2 +-sensing receptor regulates blood vessel tone and blood pressure. Am. J. Physiol. Cell Physiol. 310, C193C204. doi: 10.1152/ajpcell.00248.2015

Smith, K. A., Ayon, R. J., Tang, H., Makino, A., and Yuan, J. X. (2016). Calciumsensing receptor regulates cytosolic $[\mathrm{Ca}(2+)]$ and plays a major role in the development of pulmonary hypertension. Front. Physiol. 7:517. doi: 10.3389/ fphys.2016.00517

Sun, Y. H., Liu, M. N., Li, H., Shi, S., Zhao, Y. J., Wang, R., et al. (2006). Calciumsensing receptor induces rat neonatal ventricular cardiomyocyte apoptosis. Biochem. Biophys. Res. Commun. 350, 942-948. doi: 10.1016/j.bbrc.2006. 09.142

Tang, B., Zhang, J. G., Tan, H. Y., and Wei, X. Q. (2018). Astragaloside IV inhibits ventricular remodeling and improves fatty acid utilization in rats with chronic heart failure. Biosci. Rep. 38:BSR20171036. doi: 10.1042/BSR201 71036

Tfelt-Hansen, J., Hansen, J. L., Smajilovic, S., Terwilliger, E. F., Haunso, S., and Sheikh, S. P. (2006). Calcium receptor is functionally expressed in rat neonatal ventricular cardiomyocytes. Am. J. Physiol. Heart Circ. Physiol. 290, H1165H1171. doi: 10.1152/ajpheart.00821.2005

Wang, L. N., Wang, C., Lin, Y., Xi, Y. H., Zhang, W. H., Zhao, Y. J., et al. (2008). Involvement of calcium-sensing receptor in cardiac hypertrophyinduced by angiotensinII through calcineurin pathway in cultured neonatal rat cardiomyocytes. Biochem. Biophys. Res. Commun. 369, 584-589. doi: 10.1016/j. bbrc.2008.02.053

Xu, X. H., Xu, J., Xue, L., Cao, H. L., Liu, X., and Chen, Y. J. (2011). VEGF attenuates development from cardiac hypertrophy to heart failure after aortic stenosis through mitochondrial mediated apoptosis and cardiomyocyte proliferation. J. Cardiothorac. Surg. 6:54. doi: 10.1186/1749-8090-6-54

Yamamura, A., Guo, Q., Yamamura, H., Zimnicka, A. M., Pohl, N. M., Smith, K. A., et al. (2012). Enhanced $\mathrm{Ca}(2+)$-sensing receptor function in idiopathic pulmonary arterial hypertension. Circ. Res. 111, 469-481. doi: 10.1161/ CIRCRESAHA.112.266361
Yang, J., Wang, H. X., Zhang, Y. J., Yang, Y. H., Lu, M. L., Zhang, J., et al. (2013). Astragaloside IV attenuates inflammatory cytokines by inhibiting TLR4/NFsmall ka, CyrillicB signaling pathway in isoproterenol-induced myocardial hypertrophy. J. Ethnopharmacol. doi: 10.1016/j.jep.2013.10.017 [Epub ahead of print].

Zhang, L., Cao, S., Deng, S., Yao, G., and Yu, T. (2016). Ischemic postconditioning and pinacidil suppress calcium overload in anoxia-reoxygenation cardiomyocytes via down-regulation of the calcium-sensing receptor. PeerJ 4:e2612. doi: $10.7717 /$ peerj.2612

Zhang, S., Tang, F., Yang, Y., Lu, M., Luan, A., Zhang, J., et al. (2015). Astragaloside IV protects against isoproterenol-induced cardiac hypertrophy by regulating NF-kappaB/PGC-1alpha signaling mediated energy biosynthesis. PLoS One 10:e0118759. doi: 10.1371/journal.pone.0118759

Zhang, X., Chen, W., Li, J., Qi, S., Hong, S., Wang, Y., et al. (2018). Involvement of mitochondrial fission in calcium sensing receptor-mediated vascular smooth muscle cells proliferation during hypertension. Biochem. Biophys. Res. Commun. 495, 454-460. doi: 10.1016/j.bbrc.2017.11.048

Zhao, Y., Hou, G., Zhang, Y., Chi, J., Zhang, L., Zou, X., et al. (2011). Involvement of the calcium-sensing receptor in cyclosporin A-induced cardiomyocyte apoptosis in rats. Pharmazie 66, 968-974.

Zheng, H., Liu, J., Liu, C., Lu, F., Zhao, Y., Jin, Z., et al. (2011). Calciumsensing receptor activating phosphorylation of PKCdelta translocation on mitochondria to induce cardiomyocyte apoptosis during ischemia/reperfusion. Mol. Cell. Biochem. 358, 335-343. doi: 10.1007/s11010-011-0984-1

Conflict of Interest Statement: The authors declare that the research was conducted in the absence of any commercial or financial relationships that could be construed as a potential conflict of interest.

Copyright (c) $2018 \mathrm{Lu}$, Leng, He, Zhang, Wang and Tang. This is an open-access article distributed under the terms of the Creative Commons Attribution License (CC BY). The use, distribution or reproduction in other forums is permitted, provided the original author(s) and the copyright owner(s) are credited and that the original publication in this journal is cited, in accordance with accepted academic practice. No use, distribution or reproduction is permitted which does not comply with these terms. 